TRANSACTIONS OF THE

AMERICAN MATHEMATICAL SOCIETY

Volume 360, Number 12, December 2008, Pages 6269-6301

S 0002-9947(08)04436-X

Article electronically published on July 21, 2008

\title{
GALOIS STRUCTURE OF HOMOGENEOUS COORDINATE RINGS
}

\author{
FRAUKE M. BLEHER AND TED CHINBURG
}

\begin{abstract}
Suppose $G$ is a finite group acting on a projective scheme $X$ over a commutative Noetherian ring $R$. We study the $R G$-modules $\mathrm{H}^{0}\left(X, \mathcal{F} \otimes \mathcal{L}^{n}\right)$ when $n \geq 0$, and $\mathcal{F}$ and $\mathcal{L}$ are coherent $G$-sheaves on $X$ such that $\mathcal{L}$ is an ample line bundle. We show that the classes of these modules in the Grothendieck group $G_{0}(R G)$ of all finitely generated $R G$-modules lie in a finitely generated subgroup. Under various hypotheses, we show that there is a finite set of indecomposable $R G$-modules such that each $\mathrm{H}^{0}\left(X, \mathcal{F} \otimes \mathcal{L}^{n}\right)$ is a direct sum of these indecomposables, with multiplicities given by generalized Hilbert polynomials for $n>>0$.
\end{abstract}

\section{INTRODUCTION}

Let $G$ be a finite group, and suppose that $X$ is a projective scheme over a commutative Noetherian ring $R$ with an action of $G$ over $R$. The primary objective of coherent equivariant Riemann-Roch Theorems is to determine equivariant Euler characteristics associated to coherent $G$-sheaves $\mathcal{F}$ on $X$. This problem has motivated much of the development of equivariant $K$-theory and intersection theory, and has substantial literature (cf. [20, [31, 32, ,9], [10, 25], and their references). One application of results on Euler characteristics is to study the $R G$-modules $\mathrm{H}^{0}\left(X, \mathcal{F} \otimes \mathcal{L}^{n}\right)$ when $\mathcal{L}$ is an ample $G$-equivariant line bundle on $X$ and $n>>0$. The most precise information one would like concerning $\mathrm{H}^{0}\left(X, \mathcal{F} \otimes \mathcal{L}^{n}\right)$ would be an explicit description of this module as a direct sum of indecomposable $R G$-modules. If $R G$ is not semi-simple, this is a more subtle problem than determining Euler characteristics. In this paper we will produce such descriptions under various geometric hypotheses on $X$, and we prove some general finiteness results concerning the classes in $G_{0}(R G)$ defined by the $\mathrm{H}^{0}\left(X, \mathcal{F} \otimes \mathcal{L}^{n}\right)$ as $n$ varies. The relation of our results to some particular results in the literature will be discussed at the end of this Introduction.

To state one of our main finiteness results, let $S(X, \mathcal{F}, \mathcal{L})$ be the graded $R G$ module $\bigoplus_{n \geq 0} \mathrm{H}^{0}\left(X, \mathcal{F} \otimes \mathcal{L}^{n}\right)$. A graded $R G$-module $M=\bigoplus_{n \in \mathbb{Z}} M_{n}$ will be said to be indecomposably finite if there is a finite set $U$ of finitely generated indecomposable $R G$-modules such that each $M_{n}$ is isomorphic to the direct sum of elements

Received by the editors May 11, 2006 and, in revised form, October 26, 2006.

2000 Mathematics Subject Classification. Primary 14L30; Secondary 14C40, 13A50, 20 C05.

Key words and phrases. Group actions on schemes, Euler characteristics, homogeneous coordinate rings, Riemann-Roch Theorems, Grothendieck groups.

The first author was supported in part by NSF Grants DMS01-39737 and DMS06-51332 and NSA Grant H98230-06-1-0021. The second author was supported in part by NSF Grants DMS0070433 and DMS05-00106. 
of $U$. A weaker condition is that there is a finitely generated subgroup of the Grothendieck group $G_{0}(R G)$ of all finitely generated $R G$-modules which contains the class of $M_{n}$ for all $n$. If this is true, we will say $M$ is finitely generated in $G_{0}(R G)$.

Theorem 1.1. The graded $R G$-module $S(X, \mathcal{F}, \mathcal{L})$ is finitely generated in $G_{0}(R G)$.

It remains to consider when $S(X, \mathcal{F}, \mathcal{L})$ is indecomposably finite, and to determine explicit decompositions of the graded summands into direct sums of indecomposable $R G$-modules. In general, $S(X, \mathcal{F}, \mathcal{L})$ is not indecomposably finite, even when $R$ is a Dedekind ring (see 93.3 . A further difficulty is that $R G$ need not have the Krull-Schmidt property, so the multiplicities of indecomposables in a given $R G$ module are not well-defined. But one can still consider sufficient conditions on $X$, $\mathcal{F}$ and $\mathcal{L}$ so that the graded summands of $S(X, \mathcal{F}, \mathcal{L})$ can be constructed in the following way.

Definition 1.2. A polynomial description $\mathfrak{P}$ of a graded $R G$-module $M=\bigoplus_{n \in \mathbb{Z}} M_{n}$ consists of the following data:

(i) An integer $m>0$;

(ii) a finite set $U$ of finitely generated non-zero $R G$-modules; and

(iii) a polynomial $P_{a, T}(t) \in \mathbb{Q}[t]$ for each $T \in U$ and each integer $a$ in the range $0 \leq a<m$ with $P_{a, T}(t) \neq 0$ for at least one $a$;

satisfying the following conditions:

(a) All elements of $U$ are indecomposable;

(b) for all sufficiently large $t \in \mathbb{Z}, P_{a, T}(t)$ is an integer; and

(c) for all $t>>0$ and $T \in U$ one has $P_{a, T}(t) \geq 0$, and there is an isomorphism of $R G$-modules

$$
M_{t m+a} \cong \bigoplus_{T \in U} T^{P_{a, T}(t)} .
$$

The degree $d(\mathfrak{P})$ of this description is the maximum of the degrees of the $P_{a, T}(t)$ in $t$.

Note that if finitely generated $R G$-modules have the Krull-Schmidt property, then the polynomials $P_{a, T}(t)$ are uniquely determined if they exist.

It is useful to consider the following variant of this definition.

Definition 1.3. Let $\mathfrak{P}$ be the data specified in Definition 1.2(i)-(iii). Let $\mathcal{G}$ be a Grothendieck group of finitely generated $R G$-modules, and suppose the classes $[T]$ and $\left[M_{t m+a}\right]$ are well-defined in $\mathcal{G}$ for $T \in U$, for all $a$ and all sufficiently large $t$. We say that $\mathfrak{P}$ is a $\mathcal{G}$-polynomial description of $M$ if instead of (c) in Definition 1.2 we have

$$
\left[M_{t m+a}\right]=\sum_{T \in U} P_{a, T}(t) \cdot[T] \text { in } \mathcal{G} \text { for } t>>0
$$

where we no longer require $P_{a, T}(t) \geq 0$.

Question 1.4. When does $S(X, \mathcal{F}, \mathcal{L})$ have a polynomial description (resp. a $\mathcal{G}$ polynomial description)?

We obtain the following answer to Question 1.4 concerning $\mathcal{G}=G_{0}(R G)$. In particular, this implies Theorem 1.1. 
Theorem 1.5. There is a $G_{0}(R G)$-polynomial description of $S(X, \mathcal{F}, \mathcal{L})$ of degree bounded by $\operatorname{dim}(\operatorname{supp}(\mathcal{F}))$.

Our other results concerning Question 1.4 have to do with the following two cases: (i) the action of $G$ on $X$ is tame, and (ii) $R$ is a field.

We first assume the action of $G$ on $X$ is tame. This means that for each point $x \in X$ the order of the inertia group $I_{x}$ of $x$ is relatively prime to the residue characteristic of $x$. Let $\mathrm{CT}(R G)$ be the Grothendieck group of all finitely generated $R G$-modules which have finite projective dimension as $\mathbb{Z} G$-modules. We allow arbitrary exact sequences of $R G$-modules in defining the relations in $\mathrm{CT}(R G)$.

Theorem 1.6. Suppose the action of $G$ on $X$ is tame. Then $S(X, \mathcal{F}, \mathcal{L})$ has a $\mathrm{CT}(R G)$-polynomial description of degree bounded by $\operatorname{dim}(\operatorname{supp}(\mathcal{F}))$.

Theorem 1.7. With the assumptions of Theorem 1.6, suppose additionally that $R$ is either a field, or a complete discrete valuation ring, or the ring of integers of a number field. Assume $\mathcal{F}$ is flat as a sheaf of $R$-modules. Then $S(X, \mathcal{F}, \mathcal{L})$ is an indecomposably finite projective $R G$-module having a polynomial description. If $R$ is a field, the degree of this description is $\operatorname{dim}(\operatorname{supp}(\mathcal{F}))$; otherwise the degree is $\operatorname{dim}(\operatorname{supp}(\mathcal{F}))-1$.

We now drop the assumption that the action of $G$ on $R$ is tame, and assume that $R=k$ is a field of characteristic $p$, with $p=0$ allowed. In particular, $X$ is a projective scheme over $k$ with a $G$-action over $k, \mathcal{L}$ is an ample $G$-equivariant line bundle on $X$, and $\mathcal{F}$ is a non-zero coherent $G$-sheaf on $X$.

Definition 1.8. Let $k$ be a field, and let $M=\bigoplus_{n \in \mathbb{Z}} M_{n}$ be a graded $k G$-module. Let $P\left(M_{n}\right)$ (resp. $F\left(M_{n}\right)$ ) be a maximal projective (resp. free) summand of $M_{n}$, and define $P(M)=\bigoplus_{n \in \mathbb{Z}} P\left(M_{n}\right)$ (resp. $\left.F(M)=\bigoplus_{n \in \mathbb{Z}} F\left(M_{n}\right)\right)$.

(i) We will say $M$ has polynomial growth if there is an integer $m>0$ for which the following is true. For each integer $a$ in the range $0 \leq a<m$ there is a polynomial $Q_{a}(t) \in \mathbb{Q}[t]$ such that

$$
\operatorname{dim}_{k}\left(M_{t m+a}\right)=Q_{a}(t)
$$

for $t>>0$. Define $d(M)$ to be the maximum of the degrees of $Q_{a}(t)$ in $t$ for $0 \leq a<m$.

(ii) We will say $M$ is projective (resp. free) up to terms of degree $c \geq 0$ if $\operatorname{dim}_{k}\left(M_{n} / P\left(M_{n}\right)\right)=O\left(n^{c}\right)\left(\right.$ resp. $\left.\operatorname{dim}_{k}\left(M_{n} / F\left(M_{n}\right)\right)=O\left(n^{c}\right)\right)$.

(iii) If $P\left(M_{n}\right)=M_{n}$ (resp. $F\left(M_{n}\right)=M_{n}$ ) for $n>>0$, we will say $M$ is eventually projective (resp. eventually free).

Let $G_{p}=G$ if $p=\operatorname{char}(k)=0$, and otherwise let $G_{p}$ be a Sylow $p$-subgroup of $G$. Let $B \subset X$ (resp. $B_{p} \subset X$ ) be the ramification locus of the quotient morphism $\pi: X \rightarrow Y=X / G$ (resp. of $\left.\pi_{p}: X \rightarrow Y_{p}=X / G_{p}\right)$.

Theorem 1.9. Suppose $R=k$ is a field of characteristic $p$, with $p=0$ allowed. Define $d=\operatorname{dim}(\operatorname{supp}(\mathcal{F})), c=\operatorname{dim}(\operatorname{supp}(\mathcal{F}) \cap B)$, and $c_{p}=\operatorname{dim}\left(\operatorname{supp}(\mathcal{F}) \cap B_{p}\right)$.

(i) Then $S(X, \mathcal{F}, \mathcal{L})$ and $P(S(X, \mathcal{F}, \mathcal{L}))$ have polynomial growth, and

$$
\max \left\{c_{p}, d(P(S(X, \mathcal{F}, \mathcal{L})))\right\}=d(S(X, \mathcal{F}, \mathcal{L}))=d .
$$

(ii) If $p=0\left(\right.$ resp. $\left.\operatorname{supp}(\mathcal{F}) \cap B_{p}=\emptyset\right)$, then $S(X, \mathcal{F}, \mathcal{L})$ is projective (resp. eventually projective $)$. Otherwise, $S(X, \mathcal{F}, \mathcal{L})$ is projective up to terms of degree $c_{p}$. 
(iii) If $\operatorname{supp}(\mathcal{F}) \cap B=\emptyset$, then $S(X, \mathcal{F}, \mathcal{L})$ is eventually free. Otherwise, $S(X, \mathcal{F}, \mathcal{L})$ is free up to terms of degree $c$.

One rationale for considering how the projectivity or freeness of the graded pieces of $S(X, \mathcal{F}, \mathcal{L})$ depends on ramification loci is that this leads to a proof of the indecomposable finiteness of $S(X, \mathcal{F}, \mathcal{L})$ in some additional cases:

Corollary 1.10. Suppose that the intersection of $\operatorname{supp}(\mathcal{F})$ with the ramification locus $B_{p}$ is either empty or of dimension 0 . Then $S(X, \mathcal{F}, \mathcal{L})$ is indecomposably finite and has a polynomial description of degree $\operatorname{dim}(\operatorname{supp}(\mathcal{F}))$.

To get further results about indecomposable finiteness, we need to make some additional assumptions about $X$ and $\mathcal{F}$, respectively. We say $X$ is acyclic if $\mathrm{H}^{i}\left(X, \mathcal{O}_{X}\right)=0$ for all $i \geq 1$. For example, $X=\mathbb{P}_{k}^{N}$ is acyclic for all $N$; also all rational surfaces, Enriques surfaces and the Godeaux surface are acyclic.

Theorem 1.11. Suppose $R=k$ is a field of positive characteristic $p$, and $X$ is a smooth projective variety over $k$ having a generically free action of $G$ over $k$. Let $r$ be a positive integer, and assume one of the following hypotheses is satisfied:

(i) $\operatorname{dim}(X)=1$ and $\mathcal{F}$ is an arbitrary non-zero coherent $G$-sheaf on $X$, or

(ii) $\operatorname{dim}(X)=2, X$ is acyclic, $r$ is sufficiently divisible and $\mathcal{F}=\mathcal{O}_{X}$, or

(iii) $X=\mathbb{P}_{k}^{3}, \mathcal{L}=\mathcal{O}_{X}(1)$ and $\mathcal{F}=\mathcal{O}_{X}$.

Then $S\left(X, \mathcal{F}, \mathcal{L}^{r}\right)$ is an indecomposably finite $k G$-module which has a polynomial description of degree $\operatorname{dim}(\operatorname{supp}(\mathcal{F}))$.

Part (i) of Theorem 1.11 is immediate from Corollary 1.10. Concerning $r$ in part (ii), we prove that it suffices that $r=m_{2}^{2}(\# G) m_{1}$, where $m_{2}$ is the maximal prime to $p$ divisor of $\# G$ and $\mathcal{L}^{m_{1}}$ is a very ample line bundle on $X$ such that $\mathrm{H}^{i}\left(X, \mathcal{L}^{s m_{1}}\right)=0$ for all integers $i, s>0$. In Remark 6.2.8, we discuss the problem of improving this value of $r$. We assume $\mathcal{F}=\mathcal{O}_{X}$ in parts (ii) and (iii) so as to be able to identify global sections with elements in the function field of $X$.

We now describe the organization of the paper together with the main ingredients involved in the proofs of the above results.

In $\$ 2$ we discuss some preliminaries. In $\oint 3$ we prove Theorems 1.5 and 1.6 using $\gamma$-filtrations. We also prove Theorem 1.7 using special properties of complete discrete valuation rings and rings of integers of number fields. We thank G. Pappas for suggesting the use of $\gamma$-filtrations in proving Theorems 1.5 and 1.6. This technique for studying equivariant Euler characteristics was introduced in his paper 24. In $\$ 4$ we show that the proofs of Theorem 1.9. Corollary 1.10 and Theorem 1.11 can be reduced to the case when the base field $k$ is algebraically closed. In $\$ 5$ we prove Theorem 1.9 and Corollary 1.10 using Riemann-Roch results of Fulton [11] and of Baum, Fulton and Quart [3]. In $\$ 6$ we prove parts (ii) and (iii) of Theorem 1.11 in the following way. We show there is a splitting of certain exact cohomology sequences arising from Koszul resolutions of the structure sheaves of zero-dimensional subschemes of $X$. These splittings lead to an inductive expression for the Krull-Schmidt decomposition of the graded pieces of $S\left(X, \mathcal{O}_{X}, \mathcal{L}\right)$. To construct the splittings, we use an argument going back to Katz and Grothendieck to show that if $X$ is an acyclic smooth variety over a field of characteristic $p$, there is a closed point of $X$ fixed by a Sylow $p$-subgroup of $G$; we thank A. Tamagawa for suggesting the use of this argument. 
We now discuss some particular results in the literature which pertain directly to Question 1.4 .

This paper has been motivated in large part by a result of Karagueuzian and Symonds which we now describe. They show in [17, 18, 19, 28, 29] that if $k$ is algebraic over $\mathbb{F}_{p}$ (or $k$ is arbitrary in the case $N=2$ ), then $S\left(\mathbb{P}_{k}^{N}, \mathcal{O}_{X}, \mathcal{O}_{X}(1)\right.$ ) has a polynomial description of degree $N$. This generalizes the work of many authors 1, 13, 2. The condition that $k$ is algebraic over $\mathbb{F}_{p}$ is a significant restriction, since if $k$ is transcendental over $\mathbb{F}_{p}$, there may exist actions of $G$ which are not defined over any algebraic extension of $\mathbb{F}_{p}$ (see Remark 6.3.5). Theorem 1.11 treats the case of $\mathbb{P}_{k}^{3}$ for arbitrary $k$ as well as acyclic (e.g. rational) surfaces.

The idea of considering when $M$ is "asymptotically mostly projective" or "asymptotically mostly free", which is similar to Definition 1.8, originates in the work of Symonds in 28]. Theorem 1.9 generalizes [28, Thms. 1.1 and 1.2] by taking into account $B$ and $B_{p}$.

In [29, Symonds defines a notion of structure theorem for a graded module which is related to the polynomial descriptions appearing in Definition 1.2, After we sent him our proof of Theorem 1.5, he proved in [29] a result of a similar nature using a very different homological method.

We would like to thank C.-L. Chai, G. Pappas, P. Symonds, A. Tamagawa and M. Taylor for helpful comments.

\section{Preliminaries}

In this section we will fix some notation and hypotheses, and we recall some well-known facts about equivariant line bundles, projective embeddings and homogeneous coordinate rings.

Let $R$ be a commutative Noetherian ring, and let $X$ be a projective scheme over $R$. We suppose that $G$ is a finite group which acts on $X$ over $R$. We will say the action of $G$ on $X$ is generically free if there is an open dense $G$-stable subset of $X$ on which the action of $G$ is étale. We will say the action of $G$ on $X$ is tame if for all points $x \in X$, the order of the inertia group $I_{x} \subset G$ of $x$ is relatively prime to the residue characteristics of $x$.

Let $\mathcal{L}$ be an ample line bundle on $X$ having an action of $G$ which is compatible with the action of $G$ on $\mathcal{O}_{X}$. Such an $\mathcal{L}$ always exists, by the following argument. From a projective embedding $X \rightarrow \mathbb{P}_{R}^{M}$ over $R$ one can construct a $G$-equivariant embedding from $X$ to the product $\left(\mathbb{P}_{R}^{M}\right)^{\# G}$ over $R$ of $\# G$ copies of $\mathbb{P}_{R}^{M}$, with $G$ permuting the factors of $\left(\mathbb{P}_{R}^{M}\right)^{\# G}$. Following this by a Segre embedding leads to a projective embedding $\iota: X \rightarrow \mathbb{P}_{R}^{N}$ which is compatible with a linear action of $G$ on $\mathbb{P}_{R}^{N}$. One can then take $\mathcal{L}=\iota^{*} \mathcal{O}_{\mathbb{P}_{R}^{N}}(1)$.

Suppose $\mathcal{F}$ is a non-zero coherent $\mathcal{O}_{X}$-module having a compatible action of $G$, and define $S(X, \mathcal{F}, \mathcal{L})=\bigoplus_{n \geq 0} \mathrm{H}^{0}\left(X, \mathcal{F} \otimes \mathcal{L}^{n}\right)$.

Let $\pi: X \rightarrow Y=X / G$ be the quotient morphism. By the arguments used to show [7, Thm. 8.1], it follows that $Y$ is a quasi-projective scheme over $R$. Since $X$ is proper and finite over $Y$, this then implies that $Y$ is proper and hence a projective scheme over $R$.

Remark 2.1. Without loss of generality, we can assume that the action of $G$ on $X$ is faithful because of the following arguments. Suppose $H \leq G$ is the kernel of the action of $G$ on $X$. Then by inflation from $G / H$ to $G$, any $R(G / H)$-module $M$ is also an $R G$-module. Moreover, $M$ is simple (resp. indecomposable) as an 
$R(G / H)$-module if and only if it has the same property as an $R G$-module. Inflation from $G / H$ to $G$ also gives a natural homomorphism $G_{0}(R(G / H)) \rightarrow G_{0}(R G)$.

Suppose now that the action of $G$ on $X$ is tame over $R$. Then for all $x \in X$, the inertia group $I_{x}$ contains $H$, and thus $\# H$ is relatively prime to the characteristic of the residue field $k(x)$. Let $\mathcal{Q}$ be a prime in $\operatorname{Spec}(R)$. Since $X$ is proper over $R$, there is a point $x \in X$ that lies over $\mathcal{Q}$. So $R / \mathcal{Q}$ is a subring of $k(x)$, which implies that $\# H \notin \mathcal{Q}$. Hence multiplication by $\# H$ is an isomorphism of $R_{\mathcal{Q}}$ for all $\mathcal{Q} \in \operatorname{Spec}(R)$, and thus $\# H$ is a unit in $R$. This means that the inflation from $G / H$ to $G$ of a projective (resp. cohomologically trivial) $R(G / H)$-module is a projective (resp. cohomologically trivial) $R G$-module. Thus we have inflation homomorphisms $K_{0}(R(G / H)) \rightarrow K_{0}(R G)$ and $\mathrm{CT}(R(G / H)) \rightarrow \mathrm{CT}(R G)$.

Remark 2.2. Theorem 1.1 can also be seen in the following purely algebraic context. Suppose $G$ acts $R$-linearly on the free $R$-module $R x_{0} \oplus \cdots \oplus R x_{N}$ of rank $N+1$ over $R$. We can extend this action in a unique way to an action of $G$ by $R$-algebra automorphisms on the polynomial ring $A=R\left[x_{0}, \cdots, x_{N}\right]$. Suppose $I$ is a $G$-stable homogeneous ideal of $A$, and let $S=\bigoplus_{n \geq 0} S_{n}$ be the graded ring $A / I$, where the grading is by degree. Suppose $M$ is a graded $R G$-module such that there is an action of $S$ on $M$ which respects the gradings of $S$ and of $M$, and $G$ acts on $S$ and $M$ compatibly. Then it follows from Theorem 1.1] and [14, Thm. 2.3.1] that $M$ is finitely generated in $G_{0}(R G)$.

\section{Projective schemes over arbitrary commutative Noetherian Rings}

The objective of this section is to prove Theorems 1.5, 1.6 and 1.7, In 93.3 , we give an example in which $R$ is a Dedekind ring and $S(X, \mathcal{F}, \mathcal{L})$ is not indecomposably finite.

We assume that the ring $R$ of $\oint 2$ is an arbitrary commutative Noetherian ring and that $G$ acts faithfully on $X$ over $R$.

3.1. Euler characteristics. As usual, $G_{0}(R G)$ denotes the Grothendieck group of all finitely generated $R G$-modules. Define $\mathcal{C}$ to be the category of all finitely generated $R G$-modules which have finite projective dimension as $\mathbb{Z} G$-modules. We let $\mathrm{CT}(R G)$ be the Grothendieck group of $\mathcal{C}$ with respect to all exact sequences.

Let $X$ be a projective scheme over $R$ with an action of $G$ over $R$. Let $G_{0}(G, X)$ (resp. $K_{0}(G, X)$ ) be the Grothendieck group of all coherent (resp. coherent locally free) $\mathcal{O}_{X}$-modules $\mathcal{T}$ having a compatible action of $G$. We then have a naive Euler characteristic homomorphism

$$
\chi^{\text {naive }}: G_{0}(G, X) \rightarrow G_{0}(R G)
$$

characterized by the fact that

$$
\chi^{\text {naive }}([\mathcal{T}])=\sum_{i}(-1)^{i}\left[\mathrm{H}^{i}(X, \mathcal{T})\right] .
$$

In [4 and [5, §8] it is shown if the action of $G$ on $X$ is tame and $\mathcal{T}$ is a coherent $G$-sheaf on $X$, then the hypercohomology complex $\mathrm{H}^{\bullet}(X, \mathcal{T})$ is isomorphic in the derived category of the homotopy category of $R G$-modules to a bounded complex $P^{\bullet}=\left\{P^{i}\right\}_{i}$ of objects of $\mathcal{C}$. This leads to the existence of a refined Euler characteristic homomorphism

$$
\chi: G_{0}(G, X) \rightarrow \mathrm{CT}(R G)
$$


characterized by the fact that

$$
\chi([\mathcal{T}])=\sum_{i}(-1)^{i}\left[P^{i}\right] .
$$

In particular, the image of $\chi([\mathcal{T}])$ in $G_{0}(R G)$ is $\chi^{\text {naive }}([\mathcal{T}])$.

Lemma 3.1.1. If $\mathrm{H}^{i}(X, \mathcal{T})=0$ for $i>0$, then $\mathrm{H}^{0}(X, \mathcal{T})$ is a finitely generated $R G$ module with class $\left[\mathrm{H}^{0}(X, \mathcal{T})\right]=\chi^{\text {naive }}([\mathcal{T}])$ in $G_{0}(R G)$. If in addition, the action of $G$ on $X$ is tame, then $\mathrm{H}^{0}(X, \mathcal{T})$ is an object of $\mathcal{C}$, and $\left[\mathrm{H}^{0}(X, \mathcal{T})\right]=\chi([\mathcal{T}])$ in $\mathrm{CT}(R G)$.

As in the statements of Theorems 1.5 and 1.6, let $\mathcal{L}$ be an ample $G$-equivariant line bundle on $X$, and let $\mathcal{F}$ be a non-zero coherent $\mathcal{O}_{X}$-module with a compatible $G$-action. Let $\pi: X \rightarrow X / G=Y$ be the quotient morphism.

Lemma 3.1.2. There is an ample line bundle $\mathcal{L}_{Y}$ on $Y$ and a $G$-equivariant $\mathcal{O}_{X}$ module isomorphism between $\pi^{*} \mathcal{L}_{Y}$ and $\mathcal{L}^{(\# G)^{2}}$.

Proof. Because $\pi: X \rightarrow Y$ is finite, we can find a finite open affine cover $\left\{V_{i}\right\}_{i}$ of $Y$ such that the restriction $\left.\mathcal{L}\right|_{U_{i}}$ of $\mathcal{L}$ to $U_{i}=\pi^{-1}\left(V_{i}\right)$ is free of rank one over $\mathcal{O}_{U_{i}}$ on some generator $f_{i}$. For each $g \in G$, let $g^{*} \mathcal{L}=\mathcal{O}_{X}$ otimes $\mathcal{O}_{X, g} \mathcal{L}$ be the pullpack of $\mathcal{L}$ via the automorphism $g: X \rightarrow X$. Then $g^{*} \mathcal{L}$ is isomorphic to $\mathcal{L}$ as an $\mathcal{O}_{X}$-module via the action of $g$; this isomorphism sends $1 \otimes_{g} f_{i}$ to $g\left(f_{i}\right)$. This gives an isomorphism

$$
\mathcal{L}^{\# G} \cong \prod_{g \in G} g^{*} \mathcal{L}
$$

sending the $G$-invariant element $m_{i}=\bigotimes_{g \in G} g\left(f_{i}\right)$ of $\mathcal{L}^{\# G}$ to $\bigotimes_{g \in G}\left(1 \otimes_{g} f_{i}\right)$. The $m_{i}$ produce local generators for a line bundle $\mathcal{H}$ on $Y$ such that $\mathcal{L}^{\# G}$ and $\pi^{*} \mathcal{H}$ are isomorphic as $\mathcal{O}_{X}$-modules. From this and the action of $G$ we obtain a cohomology class in $\mathrm{H}^{1}\left(G, \operatorname{Aut}_{\mathcal{O}_{X}}\left(\mathcal{L}^{\# G}\right)\right)$. Since this group is annihilated by $\# G$, we can raise $\mathcal{L}^{\# G}$ to the $\# G^{\text {th }}$ power to make this cohomology class trivial, leading to a $G$ equivariant isomorphism between $\mathcal{L}^{(\# G)^{2}}$ and $\pi^{*} \mathcal{L}_{Y}$ when $\mathcal{L}_{Y}=\mathcal{H}^{\# G}$. To show that $\mathcal{L}_{Y}$ is ample, it suffices to show that some power of $\mathcal{L}_{Y}$ is ample. By replacing $\mathcal{L}$ by a sufficiently high power, we can assume that all of the $f_{i}$ above are global sections of $\mathcal{L}$, and that the complement $X_{f_{i}}$ of the zero locus of $f_{i}$ in $X$ is affine. Then the $m_{i}$ are global sections of $\mathcal{L}_{Y}$. Since $\pi^{-1}\left(Y_{m_{i}}\right)=\bigcap_{g \in G} X_{g\left(f_{i}\right)}$ is affine and $Y_{m_{i}}=\pi^{-1}\left(Y_{m_{i}}\right) / G$, we conclude each $Y_{m_{i}}$ is affine, so $\mathcal{L}_{Y}$ is ample on $Y$ by [16, p. $155]$.

Remark 3.1.3. We need the following combinatorial result which is a slight variant of [24, Lemma 4.8]. Suppose $f: \mathbb{Z} \rightarrow A$ is a function from $\mathbb{Z}$ to an abelian group $A$. Define the difference operator $\Delta$ on functions by $\Delta(f)(n)=f(n+1)-f(n)$. Then $\Delta^{k}(f)(n)=\sum_{\ell=0}^{k}\left(\begin{array}{l}k \\ \ell\end{array}\right)(-1)^{\ell} f(n+k-\ell)$ for all $k \geq 0$. If $n_{0}$ is a fixed integer, then $(1+\Delta)^{n-n_{0}}(f)\left(n_{0}\right)=f(n)$ for all $n \geq n_{0}$. Thus we obtain the following formula for $n \geq n_{0}$ :

$$
f(n)=\sum_{\ell=0}^{\infty}\left(\begin{array}{c}
n-n_{0} \\
\ell
\end{array}\right) \cdot \Delta^{\ell}(f)\left(n_{0}\right) .
$$

Proof of Theorem 1.5 (resp. Theorem 1.6). By replacing $X$ by the support of $\mathcal{F}$, we can assume $d=\operatorname{dim}(X)=\operatorname{dim}(\operatorname{supp}(\mathcal{F}))$. Let $A=G_{0}(R G)$ (resp. $A=$ 
$\mathrm{CT}(R G))$, and let $\Delta$ be the difference operator on functions $f: \mathbb{Z} \rightarrow A$ defined by $\Delta(f)(n)=f(n+1)-f(n)$. By Lemma 3.1.1 and Remark 3.1.3, it will suffice to show that if $0 \leq j<(\# G)^{2}$ and $f_{j}(n)=\chi^{\text {naive }}\left(\left[\mathcal{F} \otimes \mathcal{L}^{\left.j+(\# G)^{2} n\right]}\right)\right.$ (resp. $f_{j}(n)=$

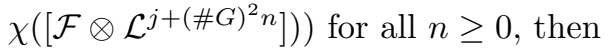

$$
\Delta^{d+1}\left(f_{j}\right)(n)=\sum_{\ell=0}^{d+1}\left(\begin{array}{c}
d+1 \\
\ell
\end{array}\right)(-1)^{\ell} f_{j}(n+d+1-\ell)=0 \text { for } n>>0
$$

when $d=\operatorname{dim}(Y)=\operatorname{dim}(X)$. For $\mathcal{L}_{Y}$ as in Lemma 3.1.2, the class $\left(\left[\mathcal{L}_{Y}\right]-1\right)^{d+1}$ lies in the $(d+1)^{\text {st }}$ term in the $\gamma$-filtration on $K_{0}(Y)=K_{0}(\{e\}, Y)$, which is trivial (see [12, Cor. V.3.10 and the Appendix to Chap. V]). Thus in $K_{0}(G, X)$ we have

$$
\begin{aligned}
0 & =[\mathcal{F}] \cdot[\mathcal{L}]^{j+(\# G)^{2} n} \cdot\left(\left[\pi^{*} \mathcal{L}_{Y}\right]-1\right)^{d+1} \\
& =[\mathcal{F}] \cdot[\mathcal{L}]^{j+(\# G)^{2} n} \cdot\left([\mathcal{L}]^{(\# G)^{2}}-1\right)^{d+1} \\
& =\sum_{\ell=0}^{d+1}\left(\begin{array}{c}
d+1 \\
\ell
\end{array}\right)(-1)^{\ell}[\mathcal{F}] \cdot[\mathcal{L}]^{j+(\# G)^{2}(n+d+1-\ell)}
\end{aligned}
$$

Taking naive Euler characteristics (resp. refined Euler characteristics) leads to (3.4) and completes the proof.

3.2. Tame actions. In this subsection we prove Theorem 1.7. We assume the action of $G$ on $X$ over $R$ is faithful and tame. We will apply the following wellknown result.

Lemma 3.2.1. Let $\mathcal{B}$ be the set of all finitely generated projective $R G$-modules, and let $\mathcal{B}_{s}$ be the set of locally free $R G$-modules of constant rank at least $s \geq 0$.

(i) Suppose $R$ is a field, a discrete valuation ring or the ring of integers of a number field. Then an object of $\mathcal{C}$ is in $\mathcal{B}$ if and only if it has no $R$-torsion.

(ii) Suppose $R$ is a discrete valuation ring. Then two elements of $\mathcal{B}$ are isomorphic if and only if they become isomorphic after tensoring with the fraction field of $R$.

(iii) Suppose $R$ is the ring of integers of a number field. Then the natural homomorphism $K_{0}(R G) \rightarrow \mathrm{CT}(R G)$ is an isomorphism, and $\mathcal{B}=\mathcal{B}_{0}$. If $P$ and $P^{\prime}$ are two elements of $\mathcal{B}_{2}$ with the same class in $\mathrm{CT}(R G)$, then $P$ and $P^{\prime}$ are isomorphic. The kernel of the rank homomorphism $r: K_{0}(R G) \rightarrow \mathbb{Z}$ is defined to be the class group $\mathrm{Cl}(R G)$ of $R G$; this is a finite abelian group. The homomorphism $\mathbb{Z} \rightarrow K_{0}(R G)$ which sends 1 to $[R G]$ splits $r$, giving an isomorphism

$$
K_{0}(R G)=\mathbb{Z} \oplus \mathrm{Cl}(R G)
$$

Proof. Part (i) follows using similar arguments as in [27, Proof of Thm. IX.5.7]. Part (ii) is [6, Thm. 32.1]. In part (iii), to prove that the natural homomorphism $K_{0}(R G) \rightarrow \mathrm{CT}(R G)$ is an isomorphism, one can use [27, Thm. IX.5.8] together with part (i). To show that two elements of $\mathcal{B}_{2}$ with the same class in $\mathrm{CT}(R G)$ are isomorphic, one can use similar arguments as in [6, Proof of Cor. 51.30]. The remainder of part (iii) can be found e.g. in [30, §1].

As in the statement of Theorem 1.7, $\mathcal{L}$ is an ample $G$-equivariant line bundle on $X$, and $\mathcal{F}$ is a non-zero coherent $\mathcal{O}_{X}$-module which is flat as a sheaf of $R$-modules and has a compatible action of $G$. To prove Theorem 1.7 we need the following result. 
Lemma 3.2.2. Let $R$ be a Dedekind ring, and suppose that $\mathcal{F}$ is flat as a sheaf of $R$-modules.

(i) The support $\operatorname{supp}(\mathcal{F})$ of $\mathcal{F}$ is flat over $\operatorname{Spec}(R)$.

(ii) Assume $R$ is the ring of integers of a number field. The $n^{\text {th }}$ graded piece $\mathrm{H}^{0}\left(X, \mathcal{F} \otimes \mathcal{L}^{n}\right)$ of $S(X, \mathcal{F}, \mathcal{L})$ is a locally free $R G$-module of rank $z(n)$, where $z(n) \rightarrow \infty$ as $n \rightarrow \infty$ unless $\operatorname{supp}(\mathcal{F})$ has dimension 1 . In the latter case, $\operatorname{supp}(\mathcal{F})$ is finite over $\operatorname{Spec}(R)$.

Proof. Since $R$ is Dedekind, to $\operatorname{prove} \operatorname{supp}(\mathcal{F})$ is flat over $R$, it will suffice to show that $\operatorname{supp}(\mathcal{F})$ is the Zariski closure of $F \otimes \operatorname{supp}(\mathcal{F})$ in $X$, where $F$ is the fraction field of $R$. Suppose $x$ is a point in $\operatorname{supp}(\mathcal{F})$ not in the Zariski closure of $F \otimes \operatorname{supp}(\mathcal{F})$. Then for each point $x^{\prime}$ of the general fiber of $X$ which has $x$ in its Zariski closure, the stalk $\mathcal{F}_{x^{\prime}}$ must be trivial. It follows that $F \otimes_{R} \mathcal{F}_{x}=\{0\}$, so $\mathcal{F}_{x}$ has non-trivial $R$-torsion, contradicting the assumption that $\mathcal{F}$ is a sheaf of flat $R$-modules. This proves part (i).

Now suppose that $R$ is the ring of integers of a number field. Since the stalks of $\mathcal{F}$ and $\mathcal{F} \otimes \mathcal{L}^{n}$ are isomorphic at each point of $X, \mathcal{F} \otimes \mathcal{L}^{n}$ is a flat sheaf of $R$-modules for all $n$. Hence $\mathrm{H}^{0}\left(X, \mathcal{F} \otimes \mathcal{L}^{n}\right)$ is flat over $R$. For large $n$, this module is cohomologically trivial for $G$ by Lemma 3.1.1. Hence by Lemma 3.2.1. $\mathrm{H}^{0}\left(X, \mathcal{F} \otimes \mathcal{L}^{n}\right)$ is a locally free $R G$-module for large $n$ of some rank $z(n) \geq 0$. On replacing $X$ by $\operatorname{supp}(\mathcal{F})$, we can suppose $X$ is flat over $R$. Suppose $\mathcal{Q} \in \operatorname{Spec}(R)$, and let $k(\mathcal{Q})$ be the residue field of $\mathcal{Q}$. Since $\mathcal{F} \otimes \mathcal{L}^{n}$ is a sheaf of flat $R$-modules, we have for $n>>0$ that

$$
z(n)=\frac{1}{\# G} \operatorname{dim}_{k(\mathcal{Q})} \mathrm{H}^{0}\left(k(\mathcal{Q}) \otimes_{R} X, k(\mathcal{Q}) \otimes_{R} \mathcal{F} \otimes \mathcal{L}^{n}\right)
$$

by considering Hilbert polynomials. Thus for large $n, z(n)$ grows as a polynomial in $n$ of degree equal to the $\operatorname{dimension} \operatorname{dim}(k(\mathcal{Q}) \otimes \operatorname{supp}(\mathcal{F}))$ of the intersection of $\operatorname{supp}(\mathcal{F})$ with the fiber of $X$ over $\mathcal{Q}$. Thus the lemma is proved unless all the fibers of $\operatorname{supp}(\mathcal{F})$ over $R$ have dimension 0 . Suppose now that the latter condition holds. Then $\operatorname{supp}(\mathcal{F})$ is quasi-finite and projective over $R$, so it is finite over $R$ (cf. [16, Ex. III.11.2]).

Proof of Theorem 1.7. By replacing $X$ by the support of $\mathcal{F}$, we can assume, by Lemma 3.2.2, that $X$ is flat over $R$ and $d=\operatorname{dim}(X)=\operatorname{dim}(\operatorname{supp}(\mathcal{F}))$. By Lemma 3.1.1. $\mathrm{H}^{0}\left(X, \mathcal{F} \otimes \mathcal{L}^{n}\right)$ is an object of $\mathcal{C}$ for large $n$; moreover, $\mathrm{H}^{0}\left(X, \mathcal{F} \otimes \mathcal{L}^{n}\right)$ has no $R$-torsion since $\mathcal{F} \otimes \mathcal{L}^{n}$ is a sheaf of flat $R$-modules for all $n$. Thus by Lemma 3.2.1, it follows that $\mathrm{H}^{0}\left(X, \mathcal{F} \otimes \mathcal{L}^{n}\right)$ is a projective $R G$-module for $n>>0$.

Suppose first that $R=F$ is a field. Since the natural map $\iota: K_{0}(F G) \rightarrow G_{0}(F G)$ is an injection, there is a $\mathbb{Q}$-linear homomorphism

$$
j: \mathbb{Q} \otimes_{\mathbb{Z}} G_{0}(F G) \rightarrow \mathbb{Q} \otimes_{\mathbb{Z}} K_{0}(F G)
$$

such that $j \circ \iota$ is the identity map when we view $K_{0}(F G)$ as a subgroup of $\mathbb{Q} \otimes_{\mathbb{Z}}$ $K_{0}(F G)$. Let $\operatorname{Irr}(F G)$ be a complete set of representatives for the isomorphism classes of simple $F G$-modules. Since $G_{0}(F G)$ is a free abelian group with basis $\operatorname{Irr}(F G)$, Theorem 1.5 implies the following. There exists an integer $m>0$ such that for each $0 \leq a<m$ and for each $V \in \operatorname{Irr}(F G)$, there exists a polynomial $Q_{a, \mathcal{F}, V}(t) \in \mathbb{Q}[t]$ of degree less than or equal to $d=\operatorname{dim}(X)$ such that for large 
$t \in \mathbb{Z}$

$$
\left[\mathrm{H}^{0}\left(X, \mathcal{F} \otimes \mathcal{L}^{t m+a}\right)\right]=\sum_{V \in \operatorname{Irr}(F G)} Q_{a, \mathcal{F}, V}(t) \cdot[V]
$$

in $G_{0}(F G)$. Since the finitely generated $F G$-modules have the Krull-Schmidt property, applying the homomorphism $j$ to this equality then shows that as $F G$-modules

$$
\mathrm{H}^{0}\left(X, \mathcal{F} \otimes \mathcal{L}^{t m+a}\right) \cong \bigoplus_{V \in \operatorname{Irr}(F G)} T_{V}^{P_{a, \mathcal{F}, T_{V}}(t)},
$$

where $T_{V}$ is the projective envelope of $V$ and $P_{a, \mathcal{F}, T_{V}}(t)$ is a $\mathbb{Q}$-linear combination of the $Q_{a, \mathcal{F}, V}(t)$ associated to $V \in \operatorname{Irr}(F G)$. Hence $S(X, \mathcal{F}, \mathcal{L})$ is an indecomposably finite $F G$-module having a polynomial description of degree bounded by $d$. Ignoring the $G$-action, we see from the Hilbert polynomial of $S(X, \mathcal{F}, \mathcal{L})$ that the degree of the description is exactly $d$.

Now let $R$ be a complete discrete valuation ring, and let $F$ be its fraction field. Then the finitely generated $R G$-modules have the Krull-Schmidt property. By Lemma 3.2.1, the map $\tau: K_{0}(R G) \rightarrow K_{0}(F G)$ induced by tensoring with $F$ over $R$ is an injection. Thus $1 \otimes \tau: \mathbb{Q} \otimes_{\mathbb{Z}} K_{0}(R G) \rightarrow \mathbb{Q} \otimes_{\mathbb{Z}} K_{0}(F G)$ has a $\mathbb{Q}$-linear section. Hence by the first paragraph of the proof, $S(X, \mathcal{F}, \mathcal{L})$ is an indecomposably finite $R G$-module having a polynomial description if and only if the same is true for $F \otimes_{R} S(X, \mathcal{F}, \mathcal{L})=S\left(F \otimes_{R} X, F \otimes_{R} \mathcal{F}, F \otimes_{R} \mathcal{L}\right)$ as an $F G$-module. The latter fact follows from the second paragraph of the proof. Since the degree of the polynomial description of $F \otimes_{R} S(X, \mathcal{F}, \mathcal{L})$ is $d-1=\operatorname{dim}\left(F \otimes_{R} X\right)$, we see that this is also the degree of the polynomial description of $S(X, \mathcal{F}, \mathcal{L})$.

Finally, let $R$ be the ring of integers of a number field with fraction field $F$. By Theorem 1.6, $S(X, \mathcal{F}, \mathcal{L})$ has a $\mathrm{CT}(R G)$-polynomial description. By Lemma 3.2.1. the quotient $\mathrm{Cl}(R G)$ of $\mathrm{CT}(R G)=K_{0}(R G)$ by the subgroup generated by the class of the free module $R G$ is a finite abelian group. Since polynomials with integer values are $\ell$-adically continuous functions for all rational primes $\ell$, the resulting $\mathrm{Cl}(R G)$-polynomial description of $S(X, \mathcal{F}, \mathcal{L})$ has the following property. There is an integer $m_{0}>0$ such that the class of the $n^{\text {th }}$ graded piece $\mathrm{H}^{0}\left(X, \mathcal{F} \otimes \mathcal{L}^{n}\right)$ of $S(X, \mathcal{F}, \mathcal{L})$ in $\mathrm{Cl}(R G)$ depends only on $n \bmod m_{0}$ for $n>>0$. Fix an integer $0 \leq a<m_{0}$. We conclude that there is a projective $R G$-module $Q_{a}$ such that for $n>>0$ and $n \equiv a \bmod m_{0}$, the class of $\mathrm{H}^{0}\left(X, \mathcal{F} \otimes \mathcal{L}^{n}\right)$ in $\mathrm{CT}(R G)=K_{0}(R G)$ equals $\left[Q_{a}\right]+\left(z(n)-r_{a}\right)[R G]$ where $z(n)\left(\right.$ resp. $\left.r_{a}\right)$ is the rank of $\mathrm{H}^{0}\left(X, \mathcal{F} \otimes \mathcal{L}^{n}\right)$ (resp. $Q_{a}$ ) as a locally free $R G$-module. Here

$$
z(n)=\frac{1}{\# G} \operatorname{dim}_{F} \mathrm{H}^{0}\left(F \otimes_{R} X,\left(F \otimes_{R} \mathcal{F}\right) \otimes\left(F \otimes_{R} \mathcal{L}^{n}\right)\right) .
$$

If $z(n) \rightarrow \infty$ as $n \rightarrow \infty$, then by Lemma 3.2.1 we conclude that $\mathrm{H}^{0}\left(X, \mathcal{F} \otimes \mathcal{L}^{n}\right)$ is isomorphic to $Q_{a} \oplus(R G)^{z(n)-r_{a}}$ as $R G$-modules for $n \equiv a \bmod m_{0}$ and $n>>0$. The rank function $z(n)$ is a polynomial in $n$ of degree equal to

$$
d-1=\operatorname{dim}\left(\operatorname{supp}\left(F \otimes_{R} \mathcal{F}\right)\right)=\operatorname{dim}\left(F \otimes_{R} X\right)
$$

for $n>>0$, so we are done in this case. In the remaining case, the support of $\mathcal{F}$ is finite over $R$ by Lemma 3.2.2. Thus, since we assumed $X=\operatorname{supp}(\mathcal{F}), X$ is flat finite and projective over $R$. Then $\mathcal{L}$ defines an element of the group $\operatorname{Pic}^{G}(X)$ of all isomorphism classes of $G$-equivariant line bundles on $X$, where the group structure of $\operatorname{Pic}^{G}(X)$ comes from the tensor product over $\mathcal{O}_{X}$. To complete the proof, it will suffice to show $\operatorname{Pic}^{G}(X)$ is finite, since then the isomorphism class of $\mathrm{H}^{0}\left(X, \mathcal{F} \otimes \mathcal{L}^{n}\right)$ 
as an $R G$-module is periodic as a function of $n$. Since $\operatorname{dim}(X)=1$ and $X$ is flat over $R, X=\operatorname{Spec}(A)$ for some $R$-order $A$ in a finite dimensional algebra $N$ over $F$. Then $\operatorname{Pic}(X)=\operatorname{Pic}(A)$ is finite since $N$ is finite dimensional over $\mathbb{Q}$. There is an injective homomorphism from the kernel of the forgetful map $\operatorname{Pic}^{G}(X) \rightarrow \operatorname{Pic}(X)$ to $\mathrm{H}^{1}\left(G, \operatorname{Aut}_{R}\left(\mathcal{O}_{X}\right)\right)$. Since $\operatorname{Aut}_{R}\left(\mathcal{O}_{X}\right)=A^{*}$ is a finitely generated abelian group, and $G$ is finite, $\mathrm{H}^{1}\left(G, \operatorname{Aut}_{R}\left(\mathcal{O}_{X}\right)\right)$ must be finite, so $\operatorname{Pic}^{G}(X)$ is as well.

3.3. A counterexample to indecomposable finiteness. Suppose $E$ is an elliptic curve over the complex numbers $\mathbb{C}$. Let $R$ be the affine ring of the complement in $E$ of the origin. Then $R$ is a Dedekind ring. Set $X=\operatorname{Spec}(R)$, and let $G$ be the trivial group. Then $\operatorname{Pic}(X)$ is isomorphic to the group of divisors of degree 0 on $E$, which is isomorphic to the group of complex points $E(\mathbb{C})$. Let $\mathcal{L}$ be a line bundle on $X$ corresponding to a point of infinite order of $E(\mathbb{C})$. Then $I=\mathrm{H}^{0}(X, \mathcal{L})$ is a locally free rank one $R$-module, and $S\left(X, \mathcal{O}_{X}, \mathcal{L}\right)=\bigoplus_{n \geq 0} \mathrm{H}^{0}\left(X, \mathcal{L}^{n}\right)=\bigoplus_{n \geq 0} I^{n}$ is not an indecomposably finite $R=R G$-module. Here $I^{n}$ denotes the $n^{\text {th }}$ power of the ideal $I$.

\section{Projective schemes over fields: \\ Reduction to ALGEBRAICALly ClOSED FIELdS}

Throughout this section we will assume the notations of 42 . Moreover, we will assume that the ring $R$ of $\oint_{2}$ is a field $k$ and that $G$ acts faithfully on $X$ over $k$. Let $G_{0}^{\oplus}(k G)$ be the Grothendieck group of all finitely generated $k G$-modules with respect to direct sums. Since $k G$ has the Krull-Schmidt property, two finitely generated $k G$-modules are isomorphic if and only if they have the same class in $G_{0}^{\oplus}(k G)$.

Define $P_{k, G}$ (resp. $N_{k, G}$ ) to be the subgroup of $G_{0}^{\oplus}(k G)$ generated by projective indecomposable (resp. non-projective indecomposable) $k G$-modules. Each of $G_{0}^{\oplus}(k G), P_{k, G}$ and $N_{k, G}$ are free abelian groups, and $G_{0}^{\oplus}(k G)=P_{k, G} \oplus N_{k, G}$. The following lemma is proved using the Krull-Schmidt Theorem.

Lemma 4.1. Let $k^{\prime}$ be an algebraic extension of $k$.

(i) The homomorphism $\psi: G_{0}^{\oplus}(k G) \rightarrow G_{0}^{\oplus}\left(k^{\prime} G\right)$ induced by tensoring with $k^{\prime}$ over $k$ is injective, and sends $P_{k, G}$ into $P_{k^{\prime}, G}$ and $N_{k, G}$ into $N_{k^{\prime}, G}$. There is a $\mathbb{Q}$-linear homomorphism $j: \mathbb{Q} \otimes_{\mathbb{Z}} G_{0}^{\oplus}\left(k^{\prime} G\right) \rightarrow \mathbb{Q} \otimes_{\mathbb{Z}} G_{0}^{\oplus}(k G)$ such that $j \circ \psi$ is the identity map.

(ii) Suppose $D$ is a finitely generated $k G$-module. If $c(k, D)$ is the codimension in $D$ of a maximal free $k G$-module summand, then $c\left(k^{\prime}, k^{\prime} \otimes_{k} D\right)=c(k, D)$.

Corollary 4.2. A graded $k G$-module $M=\bigoplus_{n \geq 0} M_{n}$ is indecomposably finite (resp. has a polynomial description of degree d, resp. is projective (resp. free) up to terms of degree c, resp. is eventually projective (resp. eventually free)) if the $k^{\prime} G$-module $k^{\prime} \otimes_{k} M$ has the corresponding property for some algebraic extension $k^{\prime}$ of $k$.

Proof. We suppose first that $k^{\prime} \otimes_{k} M$ has a polynomial description of degree $d$, and we will show $M$ has such a description; indecomposable finiteness can be treated in a similar way. There is then an integer $m>0$ and a finite set $U^{\prime}$ of finitely generated indecomposable $k^{\prime} G$-modules $T^{\prime}$ together with polynomials $P_{a, T^{\prime}}(t) \in \mathbb{Q}[t]$ such 
that for large $t \in \mathbb{Z}$, one has $P_{a, T^{\prime}}(t) \geq 0$ and

$$
k^{\prime} \otimes_{k} M_{t m+a} \cong \bigoplus_{T^{\prime} \in U^{\prime}} T^{P_{a, T^{\prime}}(t)}
$$

as $k^{\prime} G$-modules. Furthermore, the degrees of the $P_{a, T^{\prime}}(t)$ in $t$ are less than or equal to $d$ with equality for at least one $P_{a, T^{\prime}}(t)$. Let $U$ be the finite set of finitely generated indecomposable $k G$-modules $T$ which occur with a non-zero coefficient in $j\left(T^{\prime}\right) \in \mathbb{Q} \otimes_{\mathbb{Z}} G_{0}^{\oplus}(k G)$ for some $T^{\prime} \in U^{\prime}$, where $j: \mathbb{Q} \otimes_{\mathbb{Z}} G_{0}^{\oplus}\left(k^{\prime} G\right) \rightarrow \mathbb{Q} \otimes_{\mathbb{Z}} G_{0}^{\oplus}(k G)$ is the homomorphism in Lemma 4.1(i). This lemma then shows that as a $k G$-module,

$$
M_{t m+a} \cong \bigoplus_{T \in U} T^{P_{a, T}(t)},
$$

where $P_{a, T}(t)$ is a finite $\mathbb{Q}$-linear combination of the $P_{a, T^{\prime}}(t)$ associated to $T^{\prime} \in U^{\prime}$. Thus we arrive at a polynomial description of $M$ of degree $d$.

If $k^{\prime} \otimes_{k} M$ is projective (resp. free) up to terms of degree $c$ (resp. eventually projective (resp. eventually free)), then it follows from Lemma 4.1 that the same is true for $M$.

Lemma 4.3. To prove Theorems 1.9, Corollary 1.10 and Theorem 1.11, it suffices to consider the case in which $k$ is algebraically closed.

Proof. Let $k^{\prime}$ be an algebraic closure of $k$, and let $X^{\prime}=k^{\prime} \otimes_{k} X$ and $\mathcal{L}^{\prime}=k^{\prime} \otimes_{k} \mathcal{L}$.

To prove the statement Theorem[1.9 (resp. Corollary 1.10), let $\mathcal{F}^{\prime}=k^{\prime} \otimes_{k} \mathcal{F}$. By flat base change, we have $k^{\prime} \otimes_{k} \mathrm{H}^{0}\left(X, \mathcal{F} \otimes \mathcal{L}^{n}\right)=\mathrm{H}^{0}\left(X^{\prime}, \mathcal{F}^{\prime} \otimes \mathcal{L}^{\prime n}\right)$ as $k^{\prime} G$-modules for all $n$. Hence by Corollary 4.2, if Theorem 1.9 (resp. Corollary 1.10) holds for $X^{\prime}, \mathcal{L}^{\prime}$ and $\mathcal{F}^{\prime}$, then it holds for $X, \mathcal{L}$ and $\mathcal{F}$.

Suppose now that $X$ is a smooth projective variety over $k$ as in Theorem 1.11. By flat base change, we have $k^{\prime} \otimes_{k} S(X, \mathcal{L})=S\left(X^{\prime}, \mathcal{L}^{\prime}\right)$ as graded $k^{\prime} G$-modules. Let $\left\{X_{i}\right\}$ be a finite set of representatives for the $G$-orbits of irreducible components of $X^{\prime}$. Since $X^{\prime}$ is smooth over $k^{\prime}$, the $X_{i}$ are smooth projective varieties over $k^{\prime}$, and $X^{\prime}$ is the disjoint union of the $G$-translates of the $X_{i}$. For each $i$, let $H_{i}$ be the stabilizer of $X_{i}$ in $G$, and let $\mathcal{L}_{i}$ be the restriction of $\mathcal{L}^{\prime}$ to $X_{i}$. Then

$$
k^{\prime} \otimes_{k} S(X, \mathcal{L})=S\left(X^{\prime}, \mathcal{L}^{\prime}\right) \cong \bigoplus_{i} \operatorname{Ind}_{H_{i}}^{G} S\left(X_{i}, \mathcal{L}_{i}\right) .
$$

Thus, by Corollary 4.2, to prove Theorem 1.11, we can reduce to the case in which $k=k^{\prime}$.

\section{Projective SChemes over FIELdS: Results UP to High CODIMENSION}

In this section we will prove Theorem 1.9 and Corollary 1.10. Throughout this section we will assume the notation of $\$ 2$ Moreover, we will assume that the ring $R$ of 92 is a field $k$. By Lemma 4.3, we can assume $k$ is algebraically closed.

If $T$ is a finitely generated $k G$-module, we denote by $P(T)$ (resp. $F(T)$ ) a maximal projective (resp. free) summand of $T$ and by $P^{\prime}(T)$ (resp. $F^{\prime}(T)$ ) a complement for $P(T)$ (resp. $F(T)$ ) in $T$. The isomorphism classes of these modules are determined by $T$. If $M=\bigoplus_{n \in \mathbb{Z}} M_{n}$ is a graded $k G$-module, we define $\mathcal{Z}(M)=$ $\bigoplus_{n \in \mathbb{Z}} \mathcal{Z}\left(M_{n}\right)$ for $\mathcal{Z} \in\left\{P, P^{\prime}, F, F^{\prime}\right\}$.

Let $p$ be the characteristic of $k$, with $p=0$ allowed. An element $g \in G$ is $p$ regular if either $p=0$, or $p>0$ and $g$ has order prime to $p$. If $p=0$ we let $\Lambda=k$; otherwise let $\Lambda$ be a complete discrete valuation ring of characteristic 0 with residue 
field $k$ such that its fraction field is sufficiently large relative to $G$. For each finitely generated $k G$-module $T$, let $\Phi(T)$ be the ordinary character of $T$ if $p=0$ and the Brauer character of $T$ if $p>0$. Thus $\Phi(T)$ is a function from the $p$-regular elements of $G$ to $\Lambda$. Let $F$ be the fraction field of $\Lambda$. Define $G_{p}=G$ if $p=0$, and otherwise let $G_{p}$ be a Sylow $p$-subgroup of $G$.

\subsection{Module theoretic results.}

Lemma 5.1.1. Suppose $\operatorname{char}(k)=p>0$, and let $T$ be a finitely generated $k G$ module. A set $\left\{z_{i}\right\}_{i}$ of elements of $T$ is a basis for a free $k G_{p}$-module summand of $T$ if and only if the elements $\left\{\operatorname{Tr}_{G_{p}}\left(z_{i}\right)\right\}_{i}$ are linearly independent over $k$. In particular,

$$
\operatorname{dim}_{k}(P(T))=\# G_{p} \cdot \operatorname{dim}_{k}\left(\operatorname{Tr}_{G_{p}}(T)\right) .
$$

Proof. Suppose there is a relation $\sum_{i} \beta_{i} z_{i}=0$ in which the $\beta_{i}$ are elements of $k G_{p}$ which are not all 0 . We can reduce to the case in which the radical of $k G_{p}$ annihilates all of the $\beta_{i}$. But then the $\beta_{i}$ are elements of $k \operatorname{Tr}_{G_{p}}$ since $G_{p}$ is a $p$-group, contradicting the assumption that the elements $\left\{\operatorname{Tr}_{G_{p}}\left(z_{i}\right)\right\}_{i}$ are linearly independent over $k$.

Lemma 5.1.2. Suppose $M=\bigoplus_{n \in \mathbb{Z}} M_{n}$ is a graded $k G$-module in which each $M_{n}$ is finitely generated. Suppose there are integer constants $0 \leq \ell_{p} \leq \ell$ such that

(i) $\operatorname{dim}_{k}\left(P^{\prime}\left(M_{n}\right)\right)=O\left(n^{\ell_{p}}\right)$; and

(ii) for each p-regular element $g \in G$ which is not the identity element, there is a polynomial $\rho_{g}(t) \in F[t]$ of degree less than or equal to $\ell$ in $t$ such that $\Phi\left(M_{n}\right)(g)=\rho_{g}(n)$ for all sufficiently large $n$.

Then $\operatorname{dim}_{k}\left(F^{\prime}\left(M_{n}\right)\right)=O\left(n^{\ell}\right)$.

Proof. Let $V$ be a simple $k G$-module. If $p=0$, let $P_{V}=V$ and let $\Xi_{V}$ be the character of $V$. If $p>0$, let $X_{V}$ be a projective $\Lambda G$-module whose reduction modulo the maximal ideal of $\Lambda$ is the projective $k G$-cover $P_{V}$ of $V$, and let $\Xi_{V}$ be the character of the $F G$-module $F \otimes_{\Lambda} X_{V}$. For $p \geq 0$, define $G_{p^{\prime}}$ to be the set of all $p$-regular elements of $G$. By [26, $\S 2.3$ and $\S 18.3$, the multiplicity $m_{n}(V)$ of $V$ in a composition series of $M_{n}$ is

$$
\frac{1}{\# G} \sum_{g \in G_{p^{\prime}}} \Xi_{V}\left(g^{-1}\right) \Phi\left(M_{n}\right)(g)=\frac{1}{\# G} \Xi_{V}(e) \cdot \operatorname{dim}_{k}\left(M_{n}\right)+O\left(n^{\ell}\right) .
$$

Here $e$ is the identity element of $G$, and the equality follows from assumption (ii). Because of assumption (i), we see that this implies that the multiplicity $m_{n}^{\prime}(V)$ of $V$ in a composition series for $P\left(M_{n}\right)$ has the form

$$
m_{n}^{\prime}(V)=\frac{1}{\# G} \Xi_{V}(e) \cdot \operatorname{dim}_{k}\left(P\left(M_{n}\right)\right)+O\left(n^{\ell}\right) .
$$

Let $P\left(M_{n}\right)=\bigoplus_{V} P_{V}^{c_{V}(n)}$, where $V$ runs over the isomorphism classes of simple $k G$-modules. Let $r_{n}$ be the largest integer less than or equal to $\frac{1}{\# G} \operatorname{dim}_{k}\left(P\left(M_{n}\right)\right)$. Then $(k G)^{r_{n}}=\bigoplus_{V} P_{V}^{r_{n} \operatorname{dim}_{k}(V)}$. In $K_{0}(k G)$ we thus have

$$
\left[P\left(M_{n}\right)\right]-r_{n}[k G]=\sum_{V}\left(c_{V}(n)-r_{n} \operatorname{dim}_{k}(V)\right)\left[P_{V}\right]
$$


Since $\Xi_{V}(e)$ is the multiplicity of $V$ in a composition series of $k G$, the image of this class in $G_{0}(k G)$ is

$$
\sum_{V}\left(m_{n}^{\prime}(V)-\Xi_{V}(e) r_{n}\right)[V]
$$

where $m_{n}^{\prime}(V)-\Xi_{V}(e) r_{n}=O\left(n^{\ell}\right)$. Because the homomorphism $K_{0}(k G) \rightarrow G_{0}(k G)$ is an injection of finitely generated free abelian groups, this implies

$$
c_{V}(n)-r_{n} \operatorname{dim}_{k}(V)=O\left(n^{\ell}\right) .
$$

We can therefore find an integer $r_{n}^{\prime} \geq 0$ such that $0 \leq c_{V}(n)-r_{n}^{\prime} \operatorname{dim}_{k}(V)=$ $O\left(n^{\ell}\right)$. Let $F_{n}$ be a free $k G$-module of rank $r_{n}^{\prime}$. It follows that $P\left(M_{n}\right)=F_{n} \oplus F_{n}^{\prime}$ with $\operatorname{dim}_{k}\left(F_{n}^{\prime}\right)=O\left(n^{\ell}\right)$. Because of assumption (i) of the lemma, this implies $\operatorname{dim}_{k}\left(F^{\prime}\left(M_{n}\right)\right)=O\left(n^{\ell}\right)$.

5.2. Proof of parts (i) and (ii) of Theorem 1.9. As in the statement of Theorem [1.9, $X$ is a projective scheme over $k, \mathcal{L}$ is an ample $G$-equivariant line bundle on $X$, and $\mathcal{F}$ is a non-zero coherent $G$-sheaf on $X$ with $d=\operatorname{dim}(\operatorname{supp}(\mathcal{F}))$. Let $\pi: X \rightarrow Y=X / G, \pi_{p}: X \rightarrow Y_{p}=X / G_{p}$ and $\zeta: Y_{p}=X / G_{p} \rightarrow Y=X / G$ be the natural quotient morphisms. By Lemma 3.1.2 there is an ample line bundle $\mathcal{L}_{Y}$ on $Y=X / G$ and a $G$-equivariant $\mathcal{O}_{X}$-module isomorphism between $\pi^{*} \mathcal{L}_{Y}$ and $\mathcal{L}^{m}$ where $m=(\# G)^{2}$. By replacing $m$ by an appropriate multiple, we may assume that $\mathcal{L}_{Y}, \zeta^{*} \mathcal{L}_{Y}$ and $\mathcal{L}^{m} \cong \pi^{*} \mathcal{L}_{Y}$ are very ample line bundles over $k$.

The fact that $S(X, \mathcal{F}, \mathcal{L})$ has polynomial growth with $d(S(X, \mathcal{F}, \mathcal{L}))=d$ follows from the fact that the Hilbert polynomial of $\mathcal{F} \otimes \mathcal{L}^{a}$ has degree $d$ for every integer $a$. If $\operatorname{char}(k)=p=0$, then $P(S(X, \mathcal{F}, \mathcal{L}))=S(X, \mathcal{F}, \mathcal{L})$, and we are done in this case.

We may replace $X$ by the support of $\mathcal{F}$, so as to be able to assume $X=\operatorname{supp}(\mathcal{F})$ and $d=\operatorname{dim}(\operatorname{supp}(\mathcal{F}))=\operatorname{dim}(X)$. Define $B_{p}$ to be the ramification locus of the cover $\pi_{p}$, and let $c_{p}=\operatorname{dim}\left(\operatorname{supp}(\mathcal{F}) \cap B_{p}\right)=\operatorname{dim}\left(\operatorname{supp}\left(B_{p}\right)\right)$. If $B_{p}$ is empty, then $\pi: X \rightarrow Y=X / G$ is tamely ramified and $S(X, \mathcal{F}, \mathcal{L})$ is eventually projective by Lemmas 3.1.1 and 3.2.1(i). In particular, $P(S(X, \mathcal{F}, \mathcal{L}))$ has polynomial growth and $d(P(S(X, \mathcal{F}, \mathcal{L})))=d$.

In the remainder of this subsection we suppose $\operatorname{char}(k)=p>0$ and $B_{p} \neq \emptyset$. We will show that $P(S(X, \mathcal{F}, \mathcal{L}))$ has polynomial growth and that $S(X, \mathcal{F}, \mathcal{L})$ is projective up to terms of degree $c_{p}$, in the sense that

$$
\operatorname{dim}_{k}\left(P^{\prime}\left(\mathrm{H}^{0}\left(X, \mathcal{F}_{n}\right)\right)\right)=O\left(n^{c_{p}}\right)
$$

when $\mathcal{F}_{n}=\mathcal{F} \otimes \mathcal{L}^{n}$. In view of Lemma 5.1.1, (5.4) is equivalent to

$$
\operatorname{dim}_{k}\left(\mathrm{H}^{0}\left(X, \mathcal{F}_{n}\right)\right)=\# G_{p} \cdot \operatorname{dim}_{k}\left(\operatorname{Tr}_{G_{p}} \mathrm{H}^{0}\left(X, \mathcal{F}_{n}\right)\right)+O\left(n^{c_{p}}\right)
$$

The trace operator $\operatorname{Tr}_{G_{p}}$ gives an exact sequence

$$
0 \rightarrow \mathcal{K}_{n} \rightarrow\left(\pi_{p}\right)_{*}\left(\mathcal{F}_{n}\right) \rightarrow \operatorname{Tr}_{G_{p}}\left(\pi_{p}\right)_{*}\left(\mathcal{F}_{n}\right) \rightarrow 0
$$

of sheaves on $Y_{p}$, where $\mathcal{K}_{n}$ is coherent. Write $n=a+r m$ for some $0 \leq a<m$ and $r \geq 0$. Since $\mathcal{L}^{m}$ is $G$-isomorphic to $\pi^{*} \mathcal{L}_{Y}$ we find from the projection formula that $\mathcal{K}_{a+r m}=\mathcal{K}_{a} \otimes_{\mathcal{O}_{Y_{p}}}\left(\zeta^{*} \mathcal{L}_{Y}\right)^{r}$. Hence $\mathrm{H}^{1}\left(Y_{p}, \mathcal{K}_{a+r m}\right)=0$ if $r$ is sufficiently large. It follows from the cohomology of (5.6) that

$$
\begin{aligned}
\operatorname{Tr}_{G_{p}}\left(\mathrm{H}^{0}\left(X, \mathcal{F}_{n}\right)\right) & =\operatorname{Tr}_{G_{p}}\left(\mathrm{H}^{0}\left(Y_{p},\left(\pi_{p}\right)_{*}\left(\mathcal{F}_{n}\right)\right)\right) \\
& =\mathrm{H}^{0}\left(Y_{p}, \operatorname{Tr}_{G_{p}}\left(\pi_{p}\right)_{*}\left(\mathcal{F}_{n}\right)\right) \text { for } n>>0
\end{aligned}
$$


The cover $U=X-B_{p} \rightarrow V=\left(X-B_{p}\right) / G_{p}$ is an étale $G_{p^{-}}$-cover. This implies that the natural morphism $\pi_{p}^{*} \operatorname{Tr}_{G_{p}}\left(\pi_{p}\right)_{*} \mathcal{F}_{a} \rightarrow \mathcal{F}_{a}$ of sheaves on $X$ has kernel and cokernel supported on $B_{p}$. This morphism is tensored by $\mathcal{L}^{r m}$ when $a$ is replaced by $n=a+r m$. Since $\operatorname{dim}\left(B_{p}\right)=c_{p}$, we see by taking Euler characteristics and Hilbert polynomials that

$$
f_{1, a}(r)=\operatorname{dim}_{k}\left(\mathrm{H}^{0}\left(X, \mathcal{F}_{a+r m}\right)\right)-\operatorname{dim}_{k}\left(\mathrm{H}^{0}\left(X, \pi_{p}^{*} \operatorname{Tr}_{G_{p}}\left(\pi_{p}\right)_{*} \mathcal{F}_{a+r m}\right)\right)
$$

is a polynomial in $r$ of degree $\leq c_{p}$ for $r>>0$.

In view of (5.7) and (5.8), to show (5.5) we are reduced to showing that

$$
f_{2, a}(r)=\operatorname{dim}_{k}\left(\mathrm{H}^{0}\left(X, \pi_{p}^{*} \mathcal{G}_{a+r m}\right)\right)-\# G_{p} \cdot \operatorname{dim}_{k}\left(\mathrm{H}^{0}\left(Y_{p}, \mathcal{G}_{a+r m}\right)\right)
$$

is a polynomial of degree $\leq c_{p}$ in $r$ for large $r$ when $\mathcal{G}_{n}=\operatorname{Tr}_{G_{p}}\left(\pi_{p}\right)_{*} \mathcal{F}_{n}$. This will then also prove that $P(S(X, \mathcal{F}, \mathcal{L}))$ has polynomial growth.

We can now use the Riemann-Roch result of [11, Thm. 18.2] for quasi-projective schemes $Z$ over $k$. Let

$$
\tau_{Z}: G_{0}(Z) \rightarrow A_{*}(Z)_{\mathbb{Q}}
$$

be the fundamental homomorphism defined in this theorem from the Grothendieck group $G_{0}(Z)$ of all coherent sheaves on $Z$ to the rational Chow $\operatorname{ring} A_{*}(Z)_{\mathbb{Q}}$ of $Z$. (In 11], $G_{0}(Z)$ is denoted by $K_{o}(Z)$.) Define $b_{p}=B / G_{p}$, so that $V=U / G_{p}=Y_{p}-b_{p}$. From [11, Prop. 1.8], the closed immersion $i: b_{p} \rightarrow Y_{p}$ and open immersion $j: V \rightarrow Y_{p}$ give an exact sequence

$$
A_{*}\left(b_{p}\right)_{\mathbb{Q}} \stackrel{i_{*}}{\longrightarrow} A_{*}\left(Y_{p}\right)_{\mathbb{Q}} \stackrel{j^{*}}{\longrightarrow} A_{*}(V)_{\mathbb{Q}} \rightarrow 0 .
$$

Let $\pi_{p}$ stand for each of the quotient morphisms $X \rightarrow Y_{p}=X / G_{p}, U \rightarrow U / G_{p}=V$ and $B_{p} \rightarrow B / G_{p}=b_{p}$. Since $U \rightarrow V$ is an étale $G_{p}$-cover, it is smooth of relative dimension 0 , and [11, Thm. 18.2] shows that

$$
\tau_{V}\left(\left(\pi_{p}\right)_{*} \pi_{p}^{*} j^{*}\left[\mathcal{G}_{n}\right]\right)=\left(\pi_{p}\right)_{*} \pi_{p}^{*} \tau_{V}\left(j^{*}\left[\mathcal{G}_{n}\right]\right)=\# G_{p} \cdot \tau_{V}\left(j^{*}\left[\mathcal{G}_{n}\right]\right) \quad \text { in } \quad A_{*}(V)_{\mathbb{Q}}
$$

Hence (5.10) shows that

$$
\tau_{Y_{p}}\left(\left(\pi_{p}\right)_{*} \pi_{p}^{*}\left[\mathcal{G}_{n}\right]\right)-\# G_{p} \cdot \tau_{Y_{p}}\left(\left[\mathcal{G}_{n}\right]\right)=i_{*} d_{n} \quad \text { in } \quad A_{*}\left(Y_{p}\right)_{\mathbb{Q}}
$$

for some $d_{n} \in A_{*}\left(b_{p}\right)_{\mathbb{Q}}$. Let $f: Y_{p} \rightarrow \operatorname{Spec}(k)$ be the structure morphism. Then applying $f_{*}$ to both sides of (5.11) and using the Riemann-Roch result of [11, Thm. 18.2] leads to

$$
\begin{aligned}
& \operatorname{dim}_{k}\left(\mathrm{H}^{0}\left(Y_{p},\left(\pi_{p}\right)_{*} \pi_{p}^{*} \mathcal{G}_{n}\right)\right) \\
& \quad=\# G_{p} \cdot \operatorname{dim}_{k}\left(\mathrm{H}^{0}\left(Y_{p}, \mathcal{G}_{n}\right)\right)+f_{*} i_{*} d_{n} \quad \text { in } \quad A_{*}(\operatorname{Spec}(k))_{\mathbb{Q}}=\mathbb{Q}
\end{aligned}
$$

for $n>>0$.

Now write $n=a+r m$ as before. Then $\mathcal{G}_{n}=\mathcal{G}_{a+r m}=\mathcal{G}_{a} \otimes\left(\zeta^{*} \mathcal{L}_{Y}\right)^{r}$. Since $\tau_{Y_{p}}: G_{0}\left(Y_{p}\right) \rightarrow A_{*}\left(Y_{p}\right)_{\mathbb{Q}}$ is compatible with respect to cap products, we find from part (2) of [11, Thm. 18.2] that

$$
i_{*} d_{a+r m}=i_{*}\left(\operatorname{ch}_{b}\left(\mathcal{L}_{b}^{r}\right) \cdot d_{a}\right)=i_{*}\left(\sum_{j=0}^{c_{p}} \frac{c_{1}\left(\mathcal{L}_{b}\right)^{j} r^{j}}{j !} \cdot d_{a}\right),
$$

where $\mathcal{L}_{b}=i^{*} \zeta^{*} \mathcal{L}_{Y}, \operatorname{ch}_{b}$ is the Chern character associated to vector bundles on $b_{p}, c_{1}\left(\mathcal{L}_{b}\right)$ is the first Chern class of $\mathcal{L}_{b}$ and $\operatorname{dim}\left(b_{p}\right)=c_{p}$. Since $f_{*}: A_{*}\left(Y_{p}\right)_{\mathbb{Q}} \rightarrow$ $A_{*}(\operatorname{Spec}(k))_{\mathbb{Q}}$ is linear, it follows that the function $r \rightarrow f_{*} i_{*} d_{a+r m}$ is a polynomial of degree bounded by $c_{p}$ in $r$. Thus (5.12) implies (5.9), which completes the proof of (5.5) and also shows that $P(S(X, \mathcal{F}, \mathcal{L}))$ has polynomial growth. Finally, the 
equality $\max \left\{c_{p}, d(P(S(X, \mathcal{F}, \mathcal{L})))\right\}=d$ follows from the fact that $S(X, \mathcal{F}, \mathcal{L})=$ $P(S(X, \mathcal{F}, \mathcal{L})) \oplus P^{\prime}(S(X, \mathcal{F}, \mathcal{L}))$.

5.3. Proof of part (iii) of Theorem 1.9. We assume the notation of the previous two subsections. In view of Lemma 5.1.2, to complete the proof of Theorem 1.9 it will suffice to show the following. Let $g \in G$ be a $p$-regular element which is not the identity element. Define $B$ to be the ramification locus of the cover $\pi: X \rightarrow Y$, and let $c=\operatorname{dim}(\operatorname{supp}(\mathcal{F}) \cap B)$. Suppose $0 \leq a<\# G$ and that $n=a+r \# G$ for some $0 \leq r \in \mathbb{Z}$. It will suffice to show that there is a polynomial $\rho_{g, a}(t) \in F[t]$ of degree less than or equal to $c$ in $t$ such that

$$
\Phi\left(\mathrm{H}^{0}\left(X, \mathcal{F} \otimes \mathcal{L}^{a+r \# G}\right)\right)(g)=\rho_{g, a}(r) \quad \text { for } \quad r>>0 .
$$

By replacing $X$ by $\operatorname{supp}(\mathcal{F})$, we can reduce to the case in which $c=\operatorname{dim}(B)$.

We will construct $\rho_{g, a}(t)$ using a result of Baum, Fulton and Quart [3]. Let $G_{0}(G, X)$ be the Grothendieck group of coherent sheaves on $X$ having a compatible action of $G$. Let $H$ be the subgroup of $G$ generated by $g$. The subscheme $X^{H}$ of $X$ fixed by $H$ is closed and hence projective since $X$ is projective. Since $k$ is algebraically closed and $g$ is a $p$-regular element, $k H$ is the direct sum of idempotent subspaces indexed by the character group $\hat{H}=\operatorname{Hom}\left(H, k^{*}\right)$ of $H$. This gives rise to an isomorphism

$$
G_{0}\left(H, X^{H}\right)=G_{0}\left(X^{H}\right) \otimes_{\mathbb{Z}} G_{0}(k H)
$$

where $G_{0}(k H)$ is isomorphic to $\mathbb{Z} \hat{H}$. Let $\Phi_{g}: G_{0}(k H) \rightarrow \Lambda$ be the ring homomorphism which sends the class $[M]$ of a $k H$-module $M$ to $\Phi(M)(g)$. We then have a homomorphism

$$
1 \otimes \Phi_{g}: G_{0}\left(H, X^{H}\right)=G_{0}\left(X^{H}\right) \otimes_{\mathbb{Z}} G_{0}(k H) \rightarrow G_{0}\left(X^{H}\right) \otimes_{\mathbb{Z}} \Lambda .
$$

Baum, Fulton and Quart show there is a commutative diagram

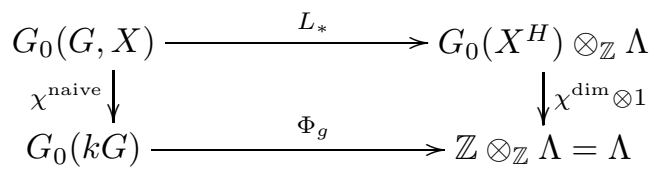

in which $\chi^{\text {naive }}: G_{0}(G, X) \rightarrow G_{0}(k G)$ (resp. $\chi^{\text {dim }}: G_{0}\left(X^{H}\right) \rightarrow \mathbb{Z}$ ) is the natural equivariant (resp. non-equivariant) Euler characteristic homomorphism, and the homomorphism $L_{*}$ has the following property. Let $K_{0}\left(H, X^{H}\right)$ be the Grothendieck group of all locally free coherent sheaves on $X^{H}$ with a compatible action of $H$. Then $K_{0}\left(H, X^{H}\right)$ is a ring via the tensor product over $\mathcal{O}_{X^{H}}$, and $G_{0}\left(H, X^{H}\right)$ is a module for this ring. For all locally free $G$-sheaves $\mathcal{E}$ on $X$ we have

$$
L_{*}([\mathcal{E} \otimes \mathcal{F}])=[\operatorname{res}(\mathcal{E})] \cdot L_{*}([\mathcal{F}]),
$$

where $[\operatorname{res}(\mathcal{E})]$ is the class in $K_{0}\left(H, X^{H}\right)$ determined by the restriction $\operatorname{res}(\mathcal{E})$ of $\mathcal{E}$ to $X^{H}$.

We now apply diagram (5.15) to the class $[\mathcal{E} \otimes \mathcal{F}] \in G_{0}(G, X)$, where $\mathcal{E}=\mathcal{L}^{n}$, $n=a+r \# G$ and $0 \leq r \in \mathbb{Z}$. The restriction $\operatorname{res}(\mathcal{L})$ of $\mathcal{L}$ to $X^{H}$ is a line bundle on $X^{H}$, with a compatible action of $H=\langle g\rangle$. Since $\operatorname{res}(\mathcal{L})$ is the direct sum of its images under the idempotents of $k H$, we conclude that for each stalk $\operatorname{res}(\mathcal{L})_{P}$ of $\operatorname{res}(\mathcal{L})$ at a point $P \in X^{H}$, all but one of these idempotents annihilates $\operatorname{res}(\mathcal{L})_{P}$, and the action of $H$ on $\operatorname{res}(\mathcal{L})_{P}$ is via a single character in $\hat{H}$. Hence $\operatorname{res}\left(\mathcal{L}^{\# G}\right)$ is a 
line bundle on $X^{H}$ with trivial $H$-action. So in $K_{0}\left(H, X^{H}\right)=K_{0}\left(X^{H}\right) \otimes_{\mathbb{Z}} G_{0}(k H)$ we have

$$
[\operatorname{res}(\mathcal{E})]=\left[\operatorname{res}\left(\mathcal{L}^{a+r \# G}\right)\right]=\left(\epsilon^{r} \otimes 1\right) \cdot\left[\operatorname{res}\left(\mathcal{L}^{a}\right)\right],
$$

where $\epsilon=\left[\operatorname{res}\left(\mathcal{L}^{\# G}\right)\right] \in K_{0}\left(X^{H}\right)$ and $\left(\epsilon^{r} \otimes 1\right) \in K_{0}\left(X^{H}\right) \otimes_{\mathbb{Z}} \Lambda$. Since $\mathcal{L}$ is ample, we have $\mathrm{H}^{i}\left(X, \mathcal{F} \otimes \mathcal{L}^{a+r \# G}\right)=0$ for all $i>0$ if $r$ is sufficiently large. Thus $\chi^{\text {naive }}([\mathcal{E} \otimes \mathcal{F}])=\left[\mathrm{H}^{0}(X, \mathcal{E} \otimes \mathcal{F})\right]$ in $G_{0}(k G)$, so

$$
\Phi_{g}\left(\chi^{\text {naive }}([\mathcal{E} \otimes \mathcal{F}])\right)=\Phi\left(\mathrm{H}^{0}\left(X, \mathcal{F} \otimes \mathcal{L}^{a+r \# G}\right)\right)(g)
$$

for $t>>0$. Hence going the other way around the diagram in (5.15) and using (5.17) gives

$$
\Phi\left(\mathrm{H}^{0}\left(X, \mathcal{F} \otimes \mathcal{L}^{a+r \# G}\right)\right)(g)=\left(\chi^{\operatorname{dim}} \otimes 1\right)\left(\left(\epsilon^{r} \otimes 1\right) \cdot\left[\operatorname{res}\left(\mathcal{L}^{a}\right)\right] \cdot L_{*}([\mathcal{F}])\right)
$$

for $r>>0$. Since $\epsilon$ is the class in $K_{0}\left(X^{H}\right)$ of the ample line bundle $\operatorname{res}\left(\mathcal{L}^{\# G}\right)$ on $X^{H}$, and $X^{H}$ is a projective scheme over $k$, the existence of Hilbert polynomials implies that the function of $r$ on the right hand side of (5.18) is for large $r$ given by a polynomial in $r$ (with coefficients in $F$ ) of degree bounded by $\operatorname{dim}\left(X^{H}\right)$. Since $H$ is not the trivial subgroup of $G, X^{H}$ is contained in the ramification locus $B$. Thus $\operatorname{dim}\left(X^{H}\right) \leq \operatorname{dim}(B)=\operatorname{dim}(B \cap \operatorname{supp}(\mathcal{F}))=c$. This shows that there is a polynomial $\rho_{a, g}(t) \in F[t]$ of the sort required in (5.13), which completes the proof.

5.4. Proof of Corollary 1.10. We assume the notation of the previous subsections. In particular, $\pi: X \rightarrow Y, \pi_{p}: X \rightarrow Y_{p}=X / G_{p}$ and $\zeta: Y_{p} \rightarrow Y$ are the natural quotient morphisms, and $B_{p}$ denotes the ramification locus of $\pi_{p}$. As before, we may reduce to the case $X=\operatorname{supp}(\mathcal{F})$. If $\operatorname{char}(k)=p=0$ or $B_{p}=\operatorname{supp}(\mathcal{F}) \cap B_{p}=\emptyset$, Corollary 1.10 follows from Theorem 1.7.

We now consider the remaining case of Corollary 1.10. Hence we suppose $\operatorname{char}(k)=p>0$ and $\operatorname{dim}\left(B_{p}\right)=\operatorname{dim}\left(\operatorname{supp}(\mathcal{F}) \cap B_{p}\right)=0$.

Lemma 5.4.1. To show Corollary 1.10, it will suffice to show that when $k$ is algebraically closed, there is an integer $m>0$ such that there is an injection for all sufficiently large $n$,

$$
P^{\prime}\left(\mathrm{H}^{0}\left(X, \mathcal{F}_{n}\right)\right) \rightarrow P^{\prime}\left(\mathrm{H}^{0}\left(X, \mathcal{F}_{n+m}\right)\right)
$$

when $\mathcal{F}_{n}=\mathcal{F} \otimes \mathcal{L}^{n}$.

Proof. By Lemma 4.3. we can reduce to the case in which $k$ is algebraically closed. Theorem 1.9 shows $\operatorname{dim}_{k}\left(P^{\prime}\left(H^{0}\left(X, \mathcal{F}_{n}\right)\right)\right)$ is bounded independently of $n$. Hence given an $m$ as in the statement of the lemma, it follows that for sufficiently large $n$, the isomorphism class of $P^{\prime}\left(H^{0}\left(X, \mathcal{F}_{n}\right)\right)$ depends only on $n \bmod m$. Hence $P^{\prime}(S(X, \mathcal{F}, \mathcal{L}))$ has a polynomial description of degree $0 . \quad$ By Theorem 1.5. $S(X, \mathcal{F}, \mathcal{L})$ has a $G_{0}(k G)$-polynomial description of degree bounded by $d$. Since

$$
S(X, \mathcal{F}, \mathcal{L})=P(S(X, \mathcal{F}, \mathcal{L})) \oplus P^{\prime}(S(X, \mathcal{F}, \mathcal{L}))
$$

it follows that $P(S(X, \mathcal{F}, \mathcal{L}))$ has a $G_{0}(k G)$-polynomial description of degree bounded by $d$. Because the terms of $P(S(X, \mathcal{F}, \mathcal{L}))$ are projective and finitely generated, and $K_{0}(k G) \rightarrow G_{0}(k G)$ is injective, we conclude that both $P(S(X, \mathcal{F}, \mathcal{L}))$ and $S(X, \mathcal{F}, \mathcal{L})$ have polynomial descriptions of degree bounded by $d$. The degrees are exactly $d$ because the ordinary Hilbert polynomial of $S(X, \mathcal{F}, \mathcal{L})$ has degree $d=\operatorname{dim}(\operatorname{supp}(\mathcal{F}))$. This implies $S(X, \mathcal{F}, \mathcal{L})$ is indecomposably finite. 
The trace operator $\operatorname{Tr}_{G_{p}}$ gives an exact sequence

$$
0 \rightarrow \operatorname{Tr}_{G_{p}}\left(\pi_{p}\right)_{*}\left(\mathcal{F}_{n}\right) \rightarrow\left(\pi_{p}\right)_{*}\left(\mathcal{F}_{n}\right) \rightarrow \mathcal{C}_{n} \rightarrow 0
$$

of sheaves on $Y_{p}$, where $\mathcal{C}_{n}$ is coherent.

Lemma 5.4.2. To produce an injection (5.19), it will suffice to show there is an integer $m>0$ and a very ample line bundle $\mathcal{L}_{Y}$ on $Y=X / G$ with the following properties:

(i) There is a $G$-equivariant $O_{X}$-module isomorphism $\mathcal{L}^{m}=\pi^{*} \mathcal{L}_{Y}$.

(ii) There is an injection $\mathcal{O}_{Y_{p}} \rightarrow \zeta^{*} \mathcal{L}_{Y}$ whose tensor product with (5.20) gives a commutative diagram

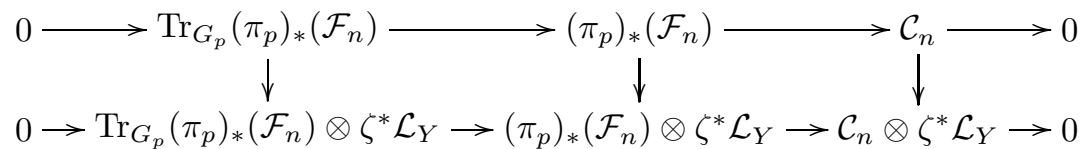

in which the vertical morphisms are injective.

Proof. The projection formula together with the fact that $\pi^{*} \mathcal{L}_{Y} \cong \mathcal{L}^{m}$ shows that the middle vertical morphism in (5.21) is a morphism

$$
\left(\pi_{p}\right)_{*}\left(\mathcal{F}_{n}\right) \rightarrow\left(\pi_{p}\right)_{*}\left(\mathcal{F}_{n}\right) \otimes \zeta^{*} \mathcal{L}_{Y} \cong\left(\pi_{p}\right)_{*}\left(\mathcal{F}_{n+m}\right) .
$$

Taking cohomology gives a morphism

$$
\tau_{n}: \mathrm{H}^{0}\left(X, \mathcal{F}_{n}\right)=\mathrm{H}^{0}\left(Y_{p},\left(\pi_{p}\right)_{*}\left(\mathcal{F}_{n}\right)\right) \rightarrow \mathrm{H}^{0}\left(Y_{p},\left(\pi_{p}\right)_{*}\left(\mathcal{F}_{n+m}\right)\right)=\mathrm{H}^{0}\left(X, \mathcal{F}_{n+m}\right) .
$$

Since (5.22) is injective by hypothesis, $\tau_{n}$ is injective. So to prove that $\tau_{n}$ gives an injection of the form in (5.19), it will suffice to show that

$$
\tau_{n}\left(P^{\prime}\left(\mathrm{H}^{0}\left(X, \mathcal{F}_{n}\right)\right)\right) \cap P\left(\mathrm{H}^{0}\left(X, \mathcal{F}_{n+m}\right)\right)=\{0\} \quad \text { for } n>>0
$$

for any choice of maximal projective summand $P\left(\mathrm{H}^{0}\left(X, \mathcal{F}_{n+m}\right)\right)$ of $\mathrm{H}^{0}\left(X, \mathcal{F}_{n+m}\right)$.

The socle of $P\left(\mathrm{H}^{0}\left(X, \mathcal{F}_{n+m}\right)\right)$ as a $k G_{p}$-module is $\operatorname{Tr}_{G_{p}} P\left(\mathrm{H}^{0}\left(X, \mathcal{F}_{n+m}\right)\right)$. Note that

$$
P^{\prime}\left(\mathrm{H}^{0}\left(X, \mathcal{F}_{n}\right)\right) \cap \operatorname{Tr}_{G_{p}} \mathrm{H}^{0}\left(X, \mathcal{F}_{n}\right)=\{0\}
$$

since $\operatorname{Tr}_{G_{p}}$ annihilates $P^{\prime}\left(\mathrm{H}^{0}\left(X, \mathcal{F}_{n}\right)\right)$. So to prove (5.23) it will suffice to show

$$
\tau_{n}\left(\mathrm{H}^{0}\left(X, \mathcal{F}_{n}\right)\right) \cap \operatorname{Tr}_{G_{p}} \mathrm{H}^{0}\left(X, \mathcal{F}_{n+m}\right)=\tau_{n}\left(\operatorname{Tr}_{G_{p}} \mathrm{H}^{0}\left(X, \mathcal{F}_{n}\right)\right) \quad \text { for } n>>0 .
$$

It was proved in (5.7) that

$$
\operatorname{Tr}_{G_{p}} \mathrm{H}^{0}\left(X, \mathcal{F}_{n}\right)=\mathrm{H}^{0}\left(Y_{p}, \operatorname{Tr}_{G_{p}}\left(\pi_{p}\right)_{*} \mathcal{F}_{n}\right) \quad \text { for } n>>0 \text {. }
$$

Hence, for $n>>0$, the cohomology over $Y_{p}$ of (5.21) gives a commutative diagram

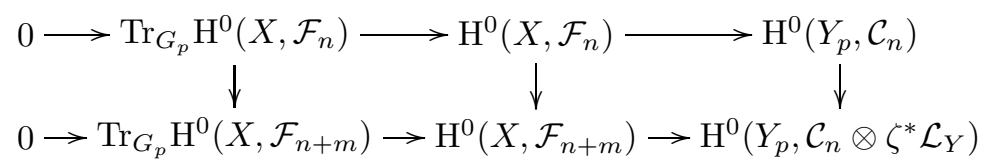

in which the vertical morphisms are induced by $\tau_{n}$. Therefore (5.24) follows from the fact that the right vertical morphism in (5.25) is injective due to the fact that the right vertical morphism in (5.21) has been assumed to be injective.

To construct a line bundle $\mathcal{L}_{Y}$ with the properties in Lemma 5.4.2, we will use the following lemma. 
Lemma 5.4.3. Suppose $Z$ is a projective scheme over an algebraically closed field $k$ and that $\mathcal{T}$ is a coherent sheaf on $Z$. There is a finite filtration of $\mathcal{T}$ by coherent sheaves such that the successive quotients are isomorphic to line bundles on a finite set $\left\{Z_{i}\right\}_{i}$ of integral closed subschemes of $Z$. Let $E$ be an effective Cartier divisor on $Z$. Then the natural morphism $\mathcal{T} \rightarrow \mathcal{T} \otimes \mathcal{O}_{Z}(E)$ is injective provided that the support of $E$ does not contain the generic point of any of the $Z_{i}$.

Proof. The first statement follows from [16, Prop. I.7.4]. Since tensoring with $\mathcal{O}_{Z}(E)$ over $\mathcal{O}_{Z}$ is exact, to prove the second statement we can reduce to the case in which $\mathcal{T}$ is a line bundle on one of the $Z_{i}$. We have an exact sequence of sheaves

$$
\operatorname{Tor}_{\mathcal{O}_{Z}}^{1}\left(\mathcal{T}, \mathcal{O}_{Z}(E) / \mathcal{O}_{Z}\right) \rightarrow \mathcal{T} \rightarrow \mathcal{T} \otimes \mathcal{O}_{Z}(E)
$$

This shows that the kernel $\mathcal{K}$ of $\mathcal{T} \rightarrow \mathcal{T} \otimes \mathcal{O}_{Z}(E)$ is annihilated by the annihilator ideal of $\mathcal{O}_{Z}(E) / \mathcal{O}_{Z}$. Hence the support of $\mathcal{K}$ is contained in the support of $E$. Since $\mathcal{K}$ is a subsheaf of the line bundle $\mathcal{T}$ on the integral scheme $Z_{i}$, the support of $\mathcal{K}$ is all of $Z_{i}$ if $\mathcal{K}$ is not 0 . However, the support of $E$ does not contain the generic point of $Z_{i}$ by assumption, so $\mathcal{K}$ must be 0 .

Completion of the proof of Corollary 1.10. By Lemma 3.1.2 there is a very ample line bundle $\mathcal{L}_{Y}$ on $Y$ such that $\pi^{*} \mathcal{L}_{Y}$ is $G$-isomorphic to $\mathcal{L}^{m}$ for some integer $m>0$. We we can use Bertini's theorem over the algebraically closed field $k$ to move $\mathcal{L}_{Y}$ so that it is defined by an effective Cartier divisor $D$ on $Y$ with the following property. Suppose $0 \leq a<m$ and that $\mathcal{T}$ is one of the sheaves $\operatorname{Tr}_{G_{p}}\left(\pi_{p}\right)_{*}\left(\mathcal{F}_{a}\right),\left(\pi_{p}\right)_{*}\left(\mathcal{F}_{a}\right)$ or $\mathcal{C}_{a}$ on $Y_{p}$. If $Z_{i}$ is an integral subscheme of $Y_{p}$ arising from a filtration of $\mathcal{T}$ of the kind described in Lemma 5.4.3, then $D$ does not contain the image in $Y$ of the generic point of $Z_{i}$. Observe that since there are only finitely many $a$ involved, these are finitely many conditions on $D$. Because $\mathcal{L}^{m} \cong \pi^{*} \mathcal{L}_{Y}$, replacing $a$ in the above condition by $a+r m$ for some $r \geq 0$ only changes each $\mathcal{T}$ by tensoring it with a power of $\zeta^{*} \mathcal{L}_{Y}$. This only changes the line bundles on the $Z_{i}$ which arise in Lemma 5.4.3. so we conclude that $D$ has the properties above when $a$ is any positive integer. We now conclude from Lemma 5.4.3 that $\mathcal{L}_{Y}$ has the properties needed in Lemma 5.4.2, which completes the proof.

\section{INDECOMPOSABLE FINITENESS \\ FOR SMOOTH PROJECTIVE VARIETIES OVER FIELDS}

The object of this section is to prove Theorem 1.11. Throughout this section we will assume the notation of 92 . Moreover, we will assume that the ring $R$ of 92 is a field $k$ of positive characteristic $p$, and that the action of $G$ on $X$ is faithful and generically free over $k$.

6.1. Line bundles and schemes of dimension 0 or 1 . By Lemma 4.3, we can assume the following hypothesis:

Hypothesis 6.1.1. The scheme $X$ is a smooth projective variety of dimension $d$ over an algebraically closed field $k$ of characteristic $p>0$, and the action of $G$ on $X$ is faithful and generically free over $k$. Let $B \subset X$ be the ramification locus of the quotient morphism $\pi: X \rightarrow Y=X / G$, and let $b$ the the image of $B$ in $Y$. The $G$-equivariant line bundle $\mathcal{L}$ on $X$ is ample.

Note that $Y=X / G$ is a Noetherian normal variety. 
Notation 6.1.2. In this section, we will write $S(X, \mathcal{L})$ for $S\left(X, \mathcal{O}_{X}, \mathcal{L}\right)$, and $\mathrm{H}^{i}(\mathcal{F})$ for $\mathrm{H}^{i}(X, \mathcal{F})$ if $\mathcal{F}$ is a coherent sheaf on $X$.

We need the following refinement of Lemma 3.1 .2

Lemma 6.1.3. Let $A$ be a finite set of closed points of $X$. Let $m_{1}$ be a positive integer such that $\mathcal{L}^{m_{1}}$ is very ample. Let $m_{2}$ be the maximal divisor of \#G which is prime to $p$. Then there exist a $G$-stable effective very ample Weil divisor $T$ on $X$ and effective Weil divisors $D_{0}, \ldots, D_{d-1}$ on $Y$ such that when $D_{j}^{\prime}=\pi^{*}\left(D_{j}\right)$ the following is true:

(i) There is a $G$-equivariant $\mathcal{O}_{X}$-module isomorphism $\psi: \mathcal{L}^{m_{1} m_{2}^{2}} \rightarrow \mathcal{O}_{X}(T)$;

(ii) for all $j, A \cap D_{j}^{\prime}=\emptyset$;

(iii) there is a $G$-equivariant $\mathcal{O}_{X}$-module isomorphism

$$
\lambda_{j}: \quad \mathcal{L}^{m_{1} m_{2}^{2}(\# G)} \rightarrow \mathcal{O}_{X}\left(D_{j}^{\prime}\right) ;
$$

(iv) the divisors $D_{0}, \ldots, D_{d-1}$ are all linearly equivalent on $Y$;

(v) for each subset $J \subset\{0, \ldots, d-1\}$ and each point $x \in \bigcap_{j \in J} D_{j}^{\prime}$, the local equations of the $D_{j}^{\prime}$ in $\mathcal{O}_{X, x}$ for $j \in J$ form a regular sequence;

(vi) the intersection $D_{0}^{\prime} \cap \cdots \cap D_{d-1}^{\prime} \cap B=\emptyset$.

Proof. Let $m_{1}$ be a positive integer such that $\mathcal{L}^{m_{1}}$ is very ample. Then $\mathrm{H}^{0}\left(\mathcal{L}^{m_{1}}\right)$ contains a non-trivial finite $G$-stable subgroup of order a power of $p$. Hence there is a non-zero element $f \in \mathrm{H}^{0}\left(\mathcal{L}^{m_{1}}\right)$ whose orbit $\omega(f)$ under the action of $G$ has order $m_{3}$ dividing $m_{2}$. The element $h=\left(\bigotimes_{\tau \in \omega(f)} \tau\right)^{m_{2} / m_{3}}$ defines a global section of $\mathcal{L}^{m_{1} m_{2}}$ which is non-zero since each $\tau \in \omega(f)$ is non-zero. Consider the $G$ equivariant embedding $X \hookrightarrow \mathbb{P}_{k}^{N^{\prime}}$ associated to $\mathrm{H}^{0}\left(\mathcal{L}^{m_{1} m_{2}}\right)$. The section $h$ defines a $G$-stable hyperplane $T^{\prime}$ in $\mathbb{P}_{k}^{N^{\prime}}$, and we let $T=T^{\prime} \cap X$. Note that since $h \neq 0$ on $X$, we ensure that $T^{\prime}$ does not contain $X$. We obtain an $\mathcal{O}_{X}$-module isomorphism $\psi_{1}: \mathcal{L}^{m_{1} m_{2}} \rightarrow \mathcal{O}_{X}(T)$. There is a $G$-action on $\operatorname{Hom}_{\mathcal{O}_{X}}\left(\mathcal{L}^{m_{1} m_{2}}, \mathcal{O}_{X}(T)\right)$ given by $(g \rho)(t)=g \rho\left(g^{-1} t\right)$ for all $\rho \in \operatorname{Hom}_{\mathcal{O}_{X}}\left(\mathcal{L}^{m_{1} m_{2}}, \mathcal{O}_{X}(T)\right)$ and all $g \in G$. Hence for all $g \in G,\left(g \psi_{1}\right): \mathcal{L}^{m_{1} m_{2}} \rightarrow \mathcal{O}_{X}(T)$ is an $\mathcal{O}_{X}$-module isomorphism. We obtain a onecocycle from $G$ to $\operatorname{Aut}_{\mathcal{O}_{X}}\left(\mathcal{O}_{X}(T)\right)$ via $g \mapsto \psi_{1} \circ\left(g \psi_{1}\right)^{-1}$. Since $k$ is algebraically closed and $X$ is a projective variety over $k$, $\operatorname{Aut}_{\mathcal{O}_{X}}\left(\mathcal{O}_{X}(T)\right)=k^{*}$. Thus the onecocycle above defines a class in $\mathrm{H}^{1}\left(G, k^{*}\right)$, where $G$ acts trivially on $k^{*}$. This cohomology class is annihilated by $m_{2}$. So replacing $\mathcal{L}^{m_{1} m_{2}}$ by $\mathcal{L}^{m_{1} m_{2}^{2}}, h$ by $h^{m_{2}}$, and $T$ by the resulting divisor on $X$, we can assume the above class in $\mathrm{H}^{1}\left(G, k^{*}\right)$ is trivial. This means that we can change $\psi_{1}$ by a scalar in $k^{*}$ so as to obtain a $G$-equivariant $\mathcal{O}_{X}$-module isomorphism $\psi: \mathcal{L}^{m_{1} m_{2}^{2}} \rightarrow \mathcal{O}_{X}(T)$, which implies (i). We let $X \hookrightarrow \mathbb{P}_{k}^{N}$ be the $G$-equivariant embedding associated to $\mathrm{H}^{0}\left(\mathcal{L}^{m_{1} m_{2}^{2}}\right)$.

Suppose $r_{0}, \ldots, r_{d-1}$ are linear forms on $\mathbb{P}_{k}^{N}$. For each $1 \leq l \leq d$ and each $l$ tuple $\left(j_{1}, \ldots, j_{l}\right)$ of distinct integers in $\{0, \ldots, d-1\}$ and each $l$-tuple $\left(g_{1}, \ldots, g_{l}\right)$ of elements of $G$, let $L\left(g_{1} r_{j_{1}}, \ldots, g_{l} r_{j_{l}}\right)$ be the common zero locus of $g_{1} r_{j_{1}}, \ldots, g_{l} r_{j_{l}}$ in $\mathbb{P}_{k}^{N}$. Define

$$
\Theta\left(g_{1} r_{j_{1}}, \ldots, g_{l} r_{j_{l}}\right)=L\left(g_{1} r_{j_{1}}, \ldots, g_{l} r_{j_{l}}\right) \cap X .
$$

For $0 \leq j \leq d-1$ let

$$
D_{j}^{\prime}=\sum_{g \in G} \Theta\left(g r_{j}\right) .
$$


Since $k$ is an algebraically closed field, we can use Bertini's Theorem to find $r_{0}, \ldots, r_{d-1}$, so the following is true:

(1) $\Theta\left(r_{j}\right) \cap \bigcup_{g \in G} g A=\emptyset$ for all $j$.

(2) $\Theta\left(r_{j}\right)$ does not contain an irreducible component of $T$ for any $j$.

(3) Fix $j \in\{0, \ldots, d-1\}$. Suppose $\left(j_{1}, \ldots, j_{l}\right)$ is an $l$-tuple of distinct integers in $\{0, \ldots, j-1\}$. Then $\Theta\left(r_{j}, g_{1} r_{j_{1}}, \ldots, g_{l} r_{j_{l}}\right)=\Theta\left(r_{j}\right) \cap \Theta\left(g_{1} r_{j_{1}}, \ldots, g_{l} r_{j_{l}}\right)$ has codimension $l+1$ in $X$ for each $l$-tuple $\left(g_{1}, \ldots, g_{l}\right)$ of elements of $G$. Moreover, $\Theta\left(r_{j}, g_{1} r_{j_{1}}, \ldots, g_{l} r_{j_{l}}\right) \cap B$ has codimension at least $l+2$ in $X$.

One applies Bertini's Theorem to show that once $r_{0}, \ldots, r_{j-1}$ have been chosen, each of the conditions in (1)-(3) is satisfied by a non-empty open dense set of linear forms $r_{j}$ in the space of all linear forms.

We now consider the rational function $\frac{\operatorname{Norm}\left(r_{j}\right)}{h^{\# G}}=\frac{\prod_{g \in G} g r_{j}}{h^{\# G}}$ on $\mathbb{P}_{k}^{N}$, which restricts to a rational function $l_{j}$ on $X$. Then $l_{j}$ is a $G$-invariant rational function in $k(Y)=k(X)^{G}$ such that $\operatorname{div}_{Y}\left(l_{j}\right)$ pulls back to

$$
\operatorname{div}_{X}\left(l_{j}\right)=D_{j}^{\prime}-\# G \cdot T \text {. }
$$

Since $Y$ is a noetherian normal variety, it is regular in codimension 1, and we define $D_{j}$ to be the Weil divisor associated to the effective part of $\operatorname{div}_{Y}\left(l_{j}\right)$. Since no irreducible component of the $G$-invariant divisor $T$ occurs in $D_{j}^{\prime}, \pi^{*}\left(D_{j}\right)=D_{j}^{\prime}$ is the zero locus of $\operatorname{Norm}\left(r_{j}\right)$ on $X$. Condition (3) implies that for each subset $J \subset\{0, \ldots, d-1\}$, the codimension of $\bigcap_{j \in J} D_{j}^{\prime}$ in $X$ is equal to \#J. By [21, Thm. 31, p. 108], this implies condition (v) in the statement of Lemma 6.1.3. Defining $\lambda_{j}=\operatorname{mult}\left(l_{j}^{-1}\right) \circ \psi^{\otimes \# G}$ leads to (iii). Conditions (ii) and (iv) are clear. For (vi) we use (3), which completes the proof.

We will make use of the following result of Nakajima to split various exact sequences of cohomology groups.

Lemma 6.1.4. Suppose that $Z$ is a scheme of dimension 0 over $k$ on which the finite group $G$ acts freely over $k$. Let $\mathcal{F}$ be a coherent $G$-sheaf on $Z$. Then $\mathrm{H}^{0}(Z, \mathcal{F})$ is a free $k G$-module.

Proof. By definition, the quotient morphism $\pi: Z \rightarrow Z / G$ is a $G$-torsor when we view $G$ as a finite constant group scheme. By descent, $\mathcal{F}$ is isomorphic to $\pi^{*}(\mathcal{H})$ for some coherent sheaf $\mathcal{H}$ on $Z / G$. By [23, Theorem 1], there is a bounded complex $C^{\bullet}$ of finitely generated free $k G$-modules such that $\mathrm{H}^{i}\left(Z, \pi^{*}(\mathcal{H})\right)=\mathrm{H}^{i}(Z, \mathcal{F})$ is isomorphic to $\mathrm{H}^{i}\left(C^{\bullet}\right)$ for all $i$. Since $Z$ has dimension 0, these groups vanish for $i \neq 0$. Since finitely generated free $k G$-modules are injective, we can split $C^{\bullet}$ completely, which implies Lemma 6.1.4

We end this subsection by observing that part (i) of Theorem 1.11 concerning curves $X$ follows from Corollary 1.10 .

6.2. Smooth projective acyclic surfaces. Let $X$ be a smooth projective acyclic surface such that $X, \mathcal{L}, Y, \pi$ and $B$ are as in Hypothesis 6.1.1] (Recall that $X$ is acyclic if $\mathrm{H}^{i}\left(\mathcal{O}_{X}\right)=0$ for all $i \geq 1$.) Using Lemma 6.1.3 we fix the following notation.

Hypothesis 6.2.1. The integer $m_{1}$ is large enough so that $\mathcal{L}^{m_{1}}$ is very ample and $\mathrm{H}^{i}\left(X, \mathcal{L}^{m_{1} s}\right)=0$ if $i, s>0$. Let $m=\# G$ and let $m_{2}$ be the maximal divisor of 
$\# G$ which is prime to $p$. Set $\mathcal{T}=\mathcal{L}^{m_{1} m_{2}^{2}}$. Let $A$ be a finite set of closed points of $X$ to be specified later. By Lemma 6.1.3, there are effective divisors $D$ and $E$ on the normal surface $Y=X / G$ with the following properties. There is a function $f \in k(Y)$ such that $\operatorname{div}_{Y}(f)=E-D$. Define $D^{\prime}=\pi^{*}(D)$ and $E^{\prime}=\pi^{*}(E)$. There are $G$-equivariant $\mathcal{O}_{X}$-module isomorphisms $\lambda: \mathcal{T}^{m} \rightarrow \mathcal{O}_{X}\left(D^{\prime}\right)={ }_{\text {def }} \mathcal{T}_{D^{\prime}}$ and $\psi: \mathcal{T} \rightarrow \mathcal{O}_{X}(T)$, where $T$ is as in Lemma 6.1.3. At each point $x \in X$ where $E^{\prime}$ and $D^{\prime}$ intersect, the local equations of these divisors in $\mathcal{O}_{X, x}$ form a regular sequence. Finally, $\emptyset=E^{\prime} \cap D^{\prime} \cap B=E^{\prime} \cap A=D^{\prime} \cap A$.

Lemma 6.2.2. Let $j$ be an integer with $0 \leq j<m=\# G$. Then for all positive integers $t$, there exists a commutative diagram of sheaves of $G-\mathcal{O}_{X}$-modules

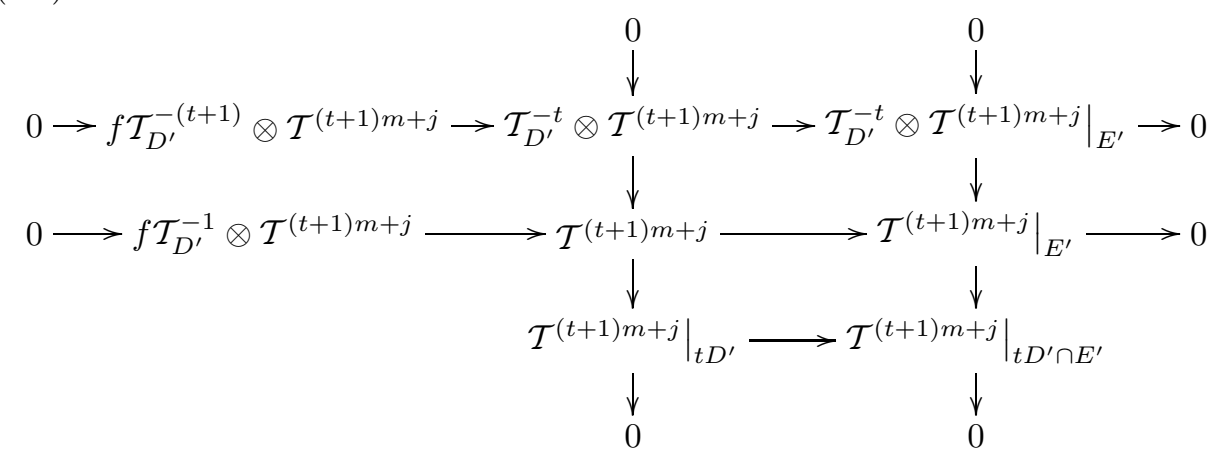

which consists of 4 short exact sequences.

Proof. The first row, resp. second row, results from tensoring the short exact sequence

$$
0 \rightarrow \mathcal{O}_{X}\left(-E^{\prime}\right) \rightarrow \mathcal{O}_{X} \rightarrow \mathcal{O}_{E^{\prime}} \rightarrow 0
$$

with $\mathcal{T}_{D^{\prime}}^{-t} \otimes \mathcal{T}^{(t+1) m+j}$, resp. with $\mathcal{T}^{(t+1) m+j}$, over $\mathcal{O}_{X}$. Note that the resulting two sequences stay exact, since $\mathcal{T}_{D^{\prime}}^{-t} \otimes \mathcal{T}^{(t+1) m+j}$, resp. $\mathcal{T}^{(t+1) m+j}$, is a line bundle on $X$, hence a flat $\mathcal{O}_{X}$-module. We obtain the middle column by tensoring the short exact sequence

$$
0 \rightarrow \mathcal{O}_{X}\left(-t D^{\prime}\right) \rightarrow \mathcal{O}_{X} \rightarrow \mathcal{O}_{t D^{\prime}} \rightarrow 0
$$

with $\mathcal{T}^{(t+1) m+j}$ over $\mathcal{O}_{X}$. As before, the resulting sequence

$$
\left.0 \rightarrow \mathcal{T}_{D^{\prime}}^{-t} \otimes \mathcal{T}^{(t+1) m+j} \rightarrow \mathcal{T}^{(t+1) m+j} \rightarrow \mathcal{T}^{(t+1) m+j}\right|_{t D^{\prime}} \rightarrow 0
$$

stays exact. For the right column, we need to show that the short exact sequence (6.2) stays exact when tensoring with $\mathcal{O}_{E^{\prime}}$, i.e. that

$$
\left.\left.\left.0 \rightarrow \mathcal{T}_{D^{\prime}}^{-t} \otimes \mathcal{T}^{(t+1) m+j}\right|_{E^{\prime}} \stackrel{\rho}{\longrightarrow} \mathcal{T}^{(t+1) m+j}\right|_{E^{\prime}} \rightarrow \mathcal{T}^{(t+1) m+j}\right|_{t D^{\prime} \cap E^{\prime}} \rightarrow 0
$$

is exact. It suffices to prove that $\rho$ is injective. Let $x \in E^{\prime} \subset X$ and let $\delta_{x}$, resp. $\epsilon_{x}$, be a local equation for $D^{\prime}$, resp. $E^{\prime}$, at $x$. Let $\gamma_{x}$ be a local generator for $\mathcal{T}$ at $x$, i.e. $\mathcal{T}_{x}=\gamma_{x} \cdot \mathcal{O}_{X, x}$. Thus, when restricting to the stalks at $x, \rho$ in (6.3) becomes

$$
\begin{gathered}
{\left[\left.\mathcal{T}_{D^{\prime}}^{-t} \otimes \mathcal{T}^{(t+1) m+j}\right|_{E^{\prime}}\right]_{x} \stackrel{\rho_{x}}{\longrightarrow}\left[\left.\mathcal{T}^{(t+1) m+j}\right|_{E^{\prime}}\right]_{x}} \\
\| \\
\frac{\delta_{x}^{t} \gamma_{x}^{\otimes((t+1) m+j)} \cdot \mathcal{O}_{X, x}}{\epsilon_{x} \cdot \delta_{x}^{t} \gamma_{x}^{\otimes((t+1) m+j)} \cdot \mathcal{O}_{X, x}}
\end{gathered}
$$


Hence $\rho_{x}$ is injective if and only if

$$
\delta_{x}^{t} \gamma_{x}^{\otimes((t+1) m+j)} \cdot \mathcal{O}_{X, x} \bigcap \epsilon_{x} \cdot \gamma_{x}^{\otimes((t+1) m+j)} \cdot \mathcal{O}_{X, x} \subseteq \epsilon_{x} \cdot \delta_{x}^{t} \gamma_{x}^{\otimes((t+1) m+j)} \cdot \mathcal{O}_{X, x} .
$$

Since $\gamma_{x}^{\otimes((t+1) m+j)} \cdot \mathcal{O}_{X, x}$ is a locally free $\mathcal{O}_{X, x}$-module and $\mathcal{O}_{X, x}$ is a unique factorization domain, to prove that $\rho_{x}$ is injective, it suffices to prove that $\delta_{x}$ and $\epsilon_{x}$ are relatively prime elements of $\mathcal{O}_{X, x}$. This is clear, since at each point $x \in E^{\prime} \cap D^{\prime}$, the local equations $\delta_{x}$ and $\epsilon_{x}$ form a regular sequence in $\mathcal{O}_{X, x}$. Hence (6.3) is exact, which proves Lemma 6.2.2

Lemma 6.2.3. Let $j$ be an integer with $0 \leq j<m=\# G$. For all positive integers $t$, we obtain from the cohomology on $X$ of the terms of the diagram (6.1) a commutative diagram of $k G$-modules

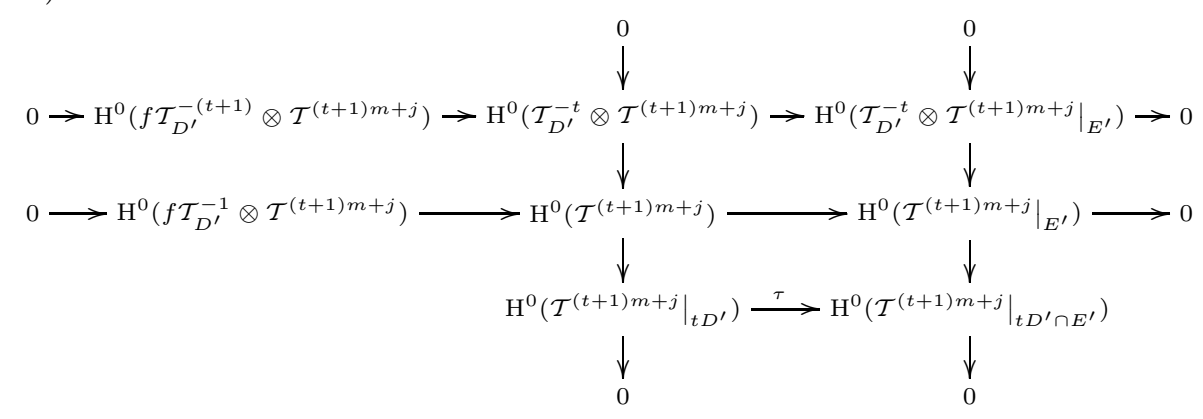

which consists of 4 short exact sequences such that the homomorphism $\tau$ is surjective.

Proof. By the Snake Lemma, it is enough to prove that the 4 sequences are exact. We use the exactness of the corresponding 4 sequences of $\mathcal{O}_{X}$-modules in (6.1). By Hypothesis 6.2.1, $\mathrm{H}^{1}\left(\mathcal{T}^{j}\right)=0$ (when $j=0$, recall that $X$ is acyclic). Hence

$$
\mathrm{H}^{1}\left(f \mathcal{T}_{D^{\prime}}^{-(t+1)} \otimes \mathcal{T}^{(t+1) m+j}\right) \cong \mathrm{H}^{1}\left(\mathcal{T}^{-(t+1) m} \otimes \mathcal{T}^{(t+1) m+j}\right)=\mathrm{H}^{1}\left(\mathcal{T}^{j}\right)=0,
$$

which gives the exactness of the first row of (6.4). Similarly,

$$
\mathrm{H}^{1}\left(f \mathcal{T}_{D^{\prime}}^{-1} \otimes \mathcal{T}^{(t+1) m+j}\right) \cong \mathrm{H}^{1}\left(\mathcal{T}^{t m+j}\right)=0
$$

and

$$
\mathrm{H}^{1}\left(\mathcal{T}_{D^{\prime}}^{-t} \otimes \mathcal{T}^{(t+1) m+j}\right) \cong \mathrm{H}^{1}\left(\mathcal{T}^{m+j}\right)=0 .
$$

Hence the second row and the middle column of (6.4) are exact. To obtain that the right column of (6.4) is exact, it suffices to show that $\mathrm{H}^{1}\left(\left.\mathcal{T}_{D^{\prime}}^{-t} \otimes \mathcal{T}^{(t+1) m+j}\right|_{E^{\prime}}\right)=0$. By taking cohomology on $X$ of the short exact sequence

$$
\left.0 \rightarrow f \mathcal{T}_{D^{\prime}}^{-(t+1)} \otimes \mathcal{T}^{(t+1) m+j} \rightarrow \mathcal{T}_{D^{\prime}}^{-t} \otimes \mathcal{T}^{(t+1) m+j} \rightarrow \mathcal{T}_{D^{\prime}}^{-t} \otimes \mathcal{T}^{(t+1) m+j}\right|_{E^{\prime}} \rightarrow 0
$$

we obtain an exact sequence

$$
\mathrm{H}^{1}\left(\mathcal{T}_{D^{\prime}}^{-t} \otimes \mathcal{T}^{(t+1) m+j}\right) \rightarrow \mathrm{H}^{1}\left(\left.\mathcal{T}_{D^{\prime}}^{-t} \otimes \mathcal{T}^{(t+1) m+j}\right|_{E^{\prime}}\right) \rightarrow \mathrm{H}^{2}\left(f \mathcal{T}_{D^{\prime}}^{-(t+1)} \otimes \mathcal{T}^{(t+1) m+j}\right) .
$$

We have $\mathrm{H}^{1}\left(\mathcal{T}_{D^{\prime}}^{-t} \otimes \mathcal{T}^{(t+1) m+j}\right) \cong \mathrm{H}^{1}\left(\mathcal{T}^{m+j}\right)=0$. Furthermore,

$$
\mathrm{H}^{2}\left(f \mathcal{T}_{D^{\prime}}^{-(t+1)} \otimes \mathcal{T}^{(t+1) m+j}\right) \cong \mathrm{H}^{2}\left(\mathcal{T}^{j}\right),
$$

and this is zero when $j=0$ since $X$ is acyclic, and zero for $j>0$ by Hypothesis 6.2.1. Hence the right column of (6.4) is exact, which completes the proof of Lemma 6.2 .3 . 
Corollary 6.2.4. Let $j$ be an integer with $0 \leq j<m$. Then for any positive integer $t$, there is an exact sequence of $k G$-modules

$$
0 \rightarrow K \rightarrow \mathrm{H}^{0}\left(\mathcal{T}^{m+j}\right) \oplus f \mathrm{H}^{0}\left(\mathcal{T}^{t m+j}\right) \rightarrow \mathrm{H}^{0}\left(\mathcal{T}^{(t+1) m+j}\right) \rightarrow Q_{t, j} \rightarrow 0,
$$

where $Q_{t, j}=\mathrm{H}^{0}\left(\left.\mathcal{T}^{(t+1) m+j}\right|_{t D^{\prime} \cap E^{\prime}}\right)$ is a free $k G$-module, and $K \cong f \mathrm{H}^{0}\left(\mathcal{T}^{j}\right)$.

Proof. Since $E^{\prime} \cap D^{\prime} \cap B=\emptyset$, it follows that $t D^{\prime} \cap E^{\prime}$ is a 0 -dimensional subvariety of $X$ with étale Galois $G$-action. Thus $Q_{t, j}$ is a free $k G$-module by Lemma 6.1.4. By Lemma 6.2.3, there is an exact sequence of $k G$-modules

$$
\mathrm{H}^{0}\left(\mathcal{T}_{D^{\prime}}^{-t} \otimes \mathcal{T}^{(t+1) m+j}\right) \oplus \mathrm{H}^{0}\left(f \mathcal{T}_{D^{\prime}}^{-1} \otimes \mathcal{T}^{(t+1) m+j}\right) \stackrel{\varphi}{\longrightarrow} \mathrm{H}^{0}\left(\mathcal{T}^{(t+1) m+j}\right) \rightarrow Q_{t, j} \rightarrow 0
$$

The $G$-equivariant $\mathcal{O}_{X}$-module isomorphism $\lambda: \mathcal{T}^{m} \rightarrow \mathcal{T}_{D^{\prime}}$ induces isomorphisms

$$
\left(\lambda^{-1}\right)^{\otimes(t+1)}: \mathcal{T}_{D^{\prime}}^{t+1-s} \otimes \mathcal{T}^{j} \rightarrow \mathcal{T}_{D^{\prime}}^{-s} \otimes \mathcal{T}^{(t+1) m+j}
$$

for all $0 \leq j<m$ and for all $1 \leq s \leq t$. We obtain that

$$
\mathrm{H}^{0}\left(\mathcal{T}_{D^{\prime}}^{-t} \otimes \mathcal{T}^{(t+1) m+j}\right)=\left(\lambda^{-1}\right)^{\otimes(t+1)} \mathrm{H}^{0}\left(\mathcal{T}_{D^{\prime}} \otimes \mathcal{T}^{j}\right)=\left(\lambda^{-1}\right)^{\otimes(t+1)} \lambda \mathrm{H}^{0}\left(\mathcal{T}^{m+j}\right)
$$

and

$$
\begin{aligned}
\mathrm{H}^{0}\left(f \mathcal{T}_{D^{\prime}}^{-1} \otimes \mathcal{T}^{(t+1) m+j}\right) & =\left(\lambda^{-1}\right)^{\otimes(t+1)} f \mathrm{H}^{0}\left(\mathcal{T}_{D^{\prime}}^{t} \otimes \mathcal{T}^{j}\right) \\
& =\left(\lambda^{-1}\right)^{\otimes(t+1)} \lambda^{\otimes t} f \mathrm{H}^{0}\left(\mathcal{T}^{t m+j}\right) .
\end{aligned}
$$

We now show that the intersection of $\mathrm{H}^{0}\left(\mathcal{T}_{D^{\prime}} \otimes \mathcal{T}^{j}\right)$ and $f \mathrm{H}^{0}\left(\mathcal{T}_{D^{\prime}}^{t} \otimes \mathcal{T}^{j}\right)$ is $f \mathrm{H}^{0}\left(\mathcal{T}^{j}\right)$ when these cohomology groups are regarded as submodules of $\mathrm{H}^{0}\left(\mathcal{T}_{D^{\prime}}^{t+1} \otimes \mathcal{T}^{j}\right)$. By Hypothesis 6.2.1 $\mathcal{T}$ is isomorphic to $\mathcal{O}_{X}(T)$, where $T$ is effective. We need to show that the intersection of $\mathrm{H}^{0}\left(\mathcal{T}_{D^{\prime}} \otimes \mathcal{O}_{X}(j T)\right)$ and $f \mathrm{H}^{0}\left(\mathcal{I}_{D^{\prime}}^{t} \otimes \mathcal{O}_{X}(j T)\right)$ is $f \mathrm{H}^{0}\left(\mathcal{O}_{X}(j T)\right)$ when these cohomology groups are regarded as submodules of $\mathrm{H}^{0}\left(\mathcal{T}_{D^{\prime}}^{t+1} \otimes \mathcal{O}_{X}(j T)\right)$. Let $h \in \mathrm{H}^{0}\left(\mathcal{T}_{D^{\prime}}^{t} \otimes \mathcal{O}_{X}(j T)\right)$ with $f h \in \mathrm{H}^{0}\left(\mathcal{T}_{D^{\prime}} \otimes \mathcal{O}_{X}(j T)\right)$. We have

$$
0 \leq \operatorname{div}_{X}(h)+t D^{\prime}+j T \text { and } 0 \leq \operatorname{div}_{X}(f h)+D^{\prime}+j T=\operatorname{div}_{X}(h)+E^{\prime}+j T .
$$

Hence $\operatorname{div}_{X}(h)+j T \geq 0$, which means that $h \in \mathrm{H}^{0}\left(\mathcal{O}_{X}(j T)\right)$. It follows that the kernel of $\varphi$ in (6.6) is $\left(\lambda^{-1}\right)^{\otimes(t+1)} f \mathrm{H}^{0}\left(\mathcal{T}^{j}\right)$, so Corollary 6.2 .4 now follows from (6.7) and (6.8).

Corollary 6.2.5. Let $j$ be an integer with $0 \leq j<m=\# G$. Then for any positive integer $t$, there is an isomorphism of $k G$-modules

$$
\mathrm{H}^{0}\left(\mathcal{T}^{(t+1) m+j}\right) \cong \frac{\mathrm{H}^{0}\left(\mathcal{O}_{X}\left(D^{\prime}+j T\right)\right)^{t+1}}{K_{t, j}} \oplus P_{t, j},
$$

where $\mathrm{H}^{0}\left(\mathcal{O}_{X}\left(D^{\prime}+j T\right)\right)^{t+1}$ is the direct sum of $t+1$ copies of $\mathrm{H}^{0}\left(\mathcal{O}_{X}\left(D^{\prime}+j T\right)\right)$,

$$
\begin{aligned}
K_{t, j}= & \mathrm{H}^{0}\left(\mathcal{O}_{X}(j T)\right) \cdot(f,-1,0,0, \ldots, 0) \oplus \mathrm{H}^{0}\left(\mathcal{O}_{X}(j T)\right) \cdot(0, f,-1,0 \ldots, 0) \\
& \oplus \cdots \oplus \mathrm{H}^{0}\left(\mathcal{O}_{X}(j T)\right) \cdot(0, \ldots, 0, f,-1)
\end{aligned}
$$

and $P_{t, j}$ is a free $k G$-module.

Proof. Let $\lambda^{\prime}=\lambda^{-1}: \mathcal{O}_{X}\left(D^{\prime}\right) \rightarrow \mathcal{T}^{m}$ and let $\psi^{\prime}=\psi^{-1}: \mathcal{O}_{X}(T) \rightarrow \mathcal{T}$. Then for all $0 \leq j<m$ and for all positive $t$, we have a $G$-equivariant $\mathcal{O}_{X}$-module homomorphism

$$
\lambda^{\prime \otimes(t+1)} \otimes \psi^{\prime \otimes j}: \mathcal{O}_{X}\left((t+1) D^{\prime}+j T\right) \rightarrow \mathcal{T}^{(t+1) m+j} .
$$


We prove by induction on $t$ that

(6.9)

$$
\begin{aligned}
& \mathrm{H}^{0}\left(\mathcal{T}^{(t+1) m+j}\right) \\
& \quad=\lambda^{\prime \otimes(t+1)} \otimes \psi^{\prime \otimes j}\left(\frac{\mathrm{H}^{0}\left(\mathcal{O}_{X}\left(D^{\prime}+j T\right)\right) \oplus \cdots \oplus \mathrm{H}^{0}\left(f^{t} \mathcal{O}_{X}\left(D^{\prime}+j T\right)\right)}{F_{t, j}} \oplus P_{t, j}\right),
\end{aligned}
$$

where

$$
\begin{aligned}
F_{t, j}= & \mathrm{H}^{0}\left(\mathcal{O}_{X}(j T)\right) \cdot(f, f \cdot(-1), 0,0, \ldots, 0) \oplus \\
& \mathrm{H}^{0}\left(\mathcal{O}_{X}(j T)\right) \cdot\left(0, f \cdot f, f^{2} \cdot(-1), 0 \ldots, 0\right) \oplus \cdots \oplus \\
& \mathrm{H}^{0}\left(\mathcal{O}_{X}(j T)\right) \cdot\left(0, \ldots, 0, f^{t-1} \cdot f, f^{t} \cdot(-1)\right) .
\end{aligned}
$$

Since $f \in k(Y)=k(X)^{G}$, there is a $k G$-module isomorphism

$$
\mathrm{H}^{0}\left(f^{l} \mathcal{O}_{X}\left(D^{\prime}+j T\right)\right) \cong \mathrm{H}^{0}\left(\mathcal{O}_{X}\left(D^{\prime}+j T\right)\right)
$$

for all $l$. Hence Corollary 6.2.5 will follow from (6.9).

When $t=1$, (6.9) follows from the proof of Corollary 6.2.4 with $P_{t, j} \cong Q_{1, j}$. For $t>1$, the proof of Corollary 6.2 .4 shows that

$$
\begin{aligned}
(6.10) & \mathrm{H}^{0}\left(\mathcal{T}^{(t+1) m+j}\right) \\
& =\lambda^{\prime \otimes(t+1)} \otimes \psi^{\prime \otimes j}\left(\frac{\mathrm{H}^{0}\left(\mathcal{O}_{X}\left(D^{\prime}+j T\right)\right) \oplus f \mathrm{H}^{0}\left(\mathcal{O}_{X}\left(t D^{\prime}+j T\right)\right)}{\left\{\mu(f, f \cdot(-1)) \mid \mu \in \mathrm{H}^{0}\left(\mathcal{O}_{X}(j T)\right)\right\}}\right) \oplus Q_{t, j} \\
& =\lambda^{\prime \otimes(t+1)} \otimes \psi^{\prime \otimes j}\left(\frac{\mathrm{H}^{0}\left(\mathcal{O}_{X}\left(D^{\prime}+j T\right)\right) \oplus\left(\psi^{\otimes j} \otimes \lambda^{\otimes t}\right) f \mathrm{H}^{0}\left(\mathcal{T}^{t m+j}\right)}{\left\{\mu(f, f \cdot(-1)) \mid \mu \in \mathrm{H}^{0}\left(\mathcal{O}_{X}(j T)\right)\right\}}\right) \oplus Q_{t, j} .
\end{aligned}
$$

By induction,

$$
\begin{aligned}
\left(\psi^{\otimes j}\right. & \left.\otimes \lambda^{\otimes t}\right) f \mathrm{H}^{0}\left(\mathcal{T}^{t m+j}\right) \\
& =f\left[\frac{\mathrm{H}^{0}\left(\mathcal{O}_{X}\left(D^{\prime}+j T\right)\right) \oplus \cdots \oplus \mathrm{H}^{0}\left(f^{t-1} \mathcal{O}_{X}\left(D^{\prime}+j T\right)\right)}{F_{t-1, j}} \oplus P_{t-1, j}\right] .
\end{aligned}
$$

Substituting (6.11) into (6.10) completes the induction and the proof.

The following theorem follows from Corollary 6.2.5.

Theorem 6.2.6. With the notation of Hypothesis 6.2.1, $S(X, \mathcal{T})$ is an indecomposably finite $k G$-module if and only if for each integer $0 \leq j<m=\# G$ there are finitely many non-isomorphic finitely generated indecomposable $k G$-modules $U(j, 1), \ldots, U\left(j, r_{j}\right)$ such that for all positive integers $t$ there exist positive integers $l_{1}(t, j), \ldots, l_{r_{j}}(t, j)$ with

$$
\frac{\mathrm{H}^{0}\left(\mathcal{O}_{X}\left(D^{\prime}+j T\right)\right)^{t+1}}{K_{t, j}} \cong U(j, 1)^{l_{1}(t, j)} \oplus \cdots \oplus U\left(j, r_{j}\right)^{l_{r_{j}}(t, j)}
$$

as $k G$-modules, where $K_{t, j}$ is as in Corollary 6.2 .5

Our goal now is to deduce from this theorem the following result, which proves part (ii) of Theorem 1.11

Theorem 6.2.7. With the notation of Hypothesis 6.2.1, $S\left(X, \mathcal{T}^{\# G}\right)$ is an indecomposably finite $k G$-module having a polynomial description of degree $d=2$. 
Remark 6.2.8. We do not know whether $S(X, \mathcal{T})$ is indecomposably finite or not. Theorem 6.2.6 shows that this only depends on the $k G$-module structure of $\mathrm{H}^{0}\left(\mathcal{O}_{X}\left(D^{\prime}+j T\right)\right)$ and on how the elements $f$ and 1 lie in $\mathrm{H}^{0}\left(\mathcal{O}_{X}\left(D^{\prime}+j T\right)\right)$ for $0 \leq j<m=\# G$.

To prove Theorem 6.2.7 we need the following result.

Lemma 6.2.9. Suppose that $Z$ is a smooth projective geometrically integral acyclic variety over a field $L$ of characteristic $p$ on which a finite p-group $G_{p}$ acts over $L$. Then there is a closed point $z_{0} \in Z$ which is fixed by $G_{p}$ as a closed subscheme of $Z$. If $L$ is finite, then $z_{0}$ can be chosen to have residue field $L$.

Proof. Suppose first that $L=\mathbb{F}_{q}$ for some power $q$ of $p$, and let $\bar{L}$ be an algebraic closure of $L$. The zeta function of $Z$ is

$$
\zeta(Z, t)=\prod_{x \in Z^{0}}\left(1-t^{\operatorname{deg}(x)}\right)^{-1},
$$

where the product is over the set $Z^{0}$ of closed points of $Z$, and $\operatorname{deg}(x)$ is the degree of $x$ over $L$. Define $\bar{Z}=\bar{L} \otimes_{L} Z$ and let $\mathbb{F}_{q}$ be the constant sheaf defined by $L$ for the étale topology of $Z$. Define $F^{*}$ to be the geometric Frobenius automorphism of $\mathrm{H}_{e t}^{n}\left(\bar{Z}, \mathbb{F}_{q}\right)$ for all $n$. By a result of Katz (see [15, XXII, 3.1] and [8, Thm 2.2]), there is a congruence

$$
\zeta(Z, t) \equiv \prod_{n \geq 0} \operatorname{det}_{\mathbb{F}_{q}}\left(1-F^{*} t \mid \mathrm{H}_{e t}^{n}\left(\bar{Z}, \mathbb{F}_{q}\right)\right)^{(-1)^{n+1}} \bmod p
$$

where on the right, one takes determinants of endomorphisms of $\mathbb{F}_{q}$-vector spaces. As in [22, 2.18(c)], we have an Artin Schreier sequence of sheaves on $Z_{e t}$,

$$
0 \longrightarrow \mathbb{F}_{q} \longrightarrow \mathbb{G}_{a} \stackrel{f-1}{\longrightarrow} \mathbb{G}_{a} \longrightarrow 0,
$$

where $f$ is the map $a \rightarrow a^{q}$. By [22, Prop. 3.7, Thm. 3.9], $\mathrm{H}_{e t}^{n}\left(\bar{Z}, \mathbb{G}_{a}\right)=\mathrm{H}^{n}\left(\bar{Z}, \mathcal{O}_{\bar{Z}}\right)$ for all $n$. Since $Z$ is acyclic and geometrically integral over the field $L=\mathbb{F}_{q}$, this implies $\mathrm{H}_{e t}^{n}\left(\bar{Z}, \mathbb{G}_{a}\right)$ is $\overline{\mathbb{F}}_{q}$ (resp. 0) if $n=0$ (resp. $n>0$ ). Taking cohomology of (6.13) over $\bar{Z}$ now shows that $\mathrm{H}_{e t}^{n}\left(\bar{Z}, \mathbb{F}_{q}\right)$ is $\mathbb{F}_{q}$ (resp. 0) if $n=0$ (resp. if $n>0$ ). Thus (6.12) gives the congruence

$$
\zeta(Z, t)=\prod_{x \in Z^{0}}\left(1-t^{\operatorname{deg}(x)}\right)^{-1} \equiv(1-t)^{-1}=\sum_{m \geq 0} t^{m} \quad \bmod p .
$$

This implies in particular that $\# Z(L)=\#\left\{x \in Z^{0}: \operatorname{deg}(x)=1\right\} \equiv 1 \bmod p$. Since $G_{p}$ is a $p$-group and permutes $Z(L)$, it has to fix a point of $Z(L)$.

If $L$ is of finite transcendence degree over $\mathbb{F}_{p}$, one chooses a quasi-projective variety $\mathcal{V}$ over $\mathbb{F}_{p}$ with function field $L$, and an integral model $\mathcal{Z}$ of $Z$ over $\mathcal{V}$ which is flat over $\mathcal{V}$. We can find a dense open subset $\mathcal{V}_{0}$ of closed points $v \in \mathcal{V}$ such that the fiber $\mathcal{Z}_{v}=\operatorname{Spec}(k(v)) \times \mathcal{Z}$ is a smooth projective geometrically integral acyclic variety over the finite residue field $k(v)$ of $v$. Since $G_{p}$ acts on $\mathcal{Z}_{v}$, the above argument shows that $\mathcal{Z}_{v}^{G_{p}}$ is non-empty. Hence the fixed point variety $\mathcal{Z}^{G_{p}}$ projects to a subset of $\mathcal{V}$ which contains a dense set of closed points. However, from [16, p. 94], we know that $\mathcal{Z}^{G_{p}}$ is closed in $\mathcal{Z}$ and has constructible image in $\mathcal{V}$, and that a constructible subset of $\mathcal{V}$ is dense if and only if it contains the generic point. We conclude that $\mathcal{Z}^{G_{p}}$ has image containing the generic point, so $Z^{G_{p}}$ is non-empty. 
Finally, for arbitrary $L$ of characteristic $p$, we use the fact that $G_{p}$ is finite to deduce that $Z$ and the $G_{p}$-action on $Z$ are defined over a field of finite transcendence degree over $\mathbb{F}_{p}$. Hence we can reduce to the previous case.

Proof of Theorem 6.2.7. Setting $j=0$ in Corollary 6.2.5 shows that for any positive integer $t$,

$$
\mathrm{H}^{0}\left(\mathcal{T}^{(t+1) m}\right) \cong \frac{\mathrm{H}^{0}\left(\mathcal{T}_{D^{\prime}}\right)^{t+1}}{K_{t}} \oplus P_{t}
$$

where

$$
K_{t}=k \cdot(f,-1,0,0, \ldots, 0) \oplus k \cdot(0, f,-1,0 \ldots, 0) \oplus \cdots \oplus k \cdot(0, \ldots, 0, f,-1)
$$

and $P_{t}$ is a free $k G$-module.

By Lemma 6.2.9, there is a closed point $x_{0} \in X$ fixed by a Sylow $p$-subgroup $G_{p}$ of $G$. In Hypothesis 6.2 .1 we let $A$ be the $G$-orbit of $x_{0}$. Thus $A \cap D^{\prime}=A \cap E^{\prime}=\emptyset$ by Hypothesis [6.2.1] so $f \in \mathcal{O}_{X, a}^{*}$ for $a \in A$ because $\operatorname{div}_{X}(f)=E^{\prime}-D^{\prime}$. Let $s(a)=\left(\mathcal{T}_{D^{\prime}}\right)_{a} /\left(\mathfrak{m}_{X, a} \cdot\left(\mathcal{T}_{D^{\prime}}\right)_{a}\right) \cong k$ for $a \in A$. Let $H$ be the inertia group of $x_{0}$, so that $G_{p} \subset H$. Consider the natural $k G$-module homomorphism

$$
k f \rightarrow \bigoplus_{a \in A} s(a)=k G \otimes_{k H} s\left(x_{0}\right)
$$

induced by taking the image of $f$ in the stalks of $\mathcal{T}_{D^{\prime}}$. Since the image of $f$ in $s\left(x_{0}\right) \cong$ $k$ is non-trivial and since $k f$ is $(G, H)$-injective by [6, Thm. 19.2 and Prop. 19.5], we find that (6.15) is split by a $k G$-module homomorphism $\tau: \bigoplus_{a \in A} s(a) \rightarrow k f$. Let $\nu: \mathrm{H}^{0}\left(\mathcal{T}_{D^{\prime}}\right) \rightarrow k f$ be the composition of the natural $k G$-module homomorphism $\mathrm{H}^{0}\left(\mathcal{T}_{D^{\prime}}\right) \rightarrow \bigoplus_{a \in A} s(a)$ with $\tau$. Define $U$ to be the kernel of $\nu$. Then $k \cdot f$ is a $k G$-complement of $U$ in $\mathrm{H}^{0}\left(\mathcal{T}_{D^{\prime}}\right)$, and $\mathrm{H}^{0}\left(\mathcal{T}_{D^{\prime}}\right)=U \oplus k f$ as $k G$-modules.

Let $U_{t}=U^{t} \oplus \mathrm{H}^{0}\left(\mathcal{T}_{D^{\prime}}\right)$, considered as a $k G$-submodule of $\mathrm{H}^{0}\left(\mathcal{T}_{D^{\prime}}\right)^{t+1}$, and suppose that

$$
\left(f_{1}, \ldots, f_{t}, f_{t+1}\right) \in K_{t} \cap U_{t} .
$$

Then there exist elements $a_{1}, \ldots, a_{t} \in K$ such that $\left(f_{1}, \ldots, f_{t}, f_{t+1}\right)=$ $\left(a_{1} f, a_{2} f-a_{1}, \ldots, a_{t} f-a_{t-1},-a_{t}\right) \in U_{t}$. Therefore $a_{1} f, a_{2} f-a_{1}, \ldots, a_{t} f-a_{t-1}$ are elements of $U$. Since $U \cap k f=0$, it follows that $a_{1}=0=a_{2}=\cdots=a_{t-1}=a_{t}$. Hence $K_{t} \cap U_{t}=0$. On the other hand, since the $k$-dimension of $\mathrm{H}^{0}\left(\mathcal{T}_{D^{\prime}}\right)$ is $\operatorname{dim}_{k}(U)+1$, it follows that $\operatorname{dim}_{k}\left(K_{t}\right)+\operatorname{dim}_{k}\left(U_{t}\right)=(t+1) \operatorname{dim}_{k}\left(\mathrm{H}^{0}\left(\mathcal{T}_{D^{\prime}}\right)\right)$. Hence

$$
\frac{\mathrm{H}^{0}\left(\mathcal{T}_{D^{\prime}}\right)^{t+1}}{K_{t}} \cong U_{t}=U^{t} \oplus \mathrm{H}^{0}\left(\mathcal{T}_{D^{\prime}}\right)
$$

as $k G$-modules. This and (6.14) give a $k G$-module isomorphism

$$
\mathrm{H}^{0}\left(\mathcal{T}^{(t+1) m}\right) \cong U^{t} \oplus \mathrm{H}^{0}\left(\mathcal{T}_{D^{\prime}}\right) \oplus P_{t}
$$

where $P_{t}$ is a free $k G$-module. Since $\operatorname{dim}_{k}\left(\mathrm{H}^{0}\left(\mathcal{T}^{(t+1) m}\right)\right)$ grows as a quadratic polynomial in $t$ for large $t$, this implies $S\left(X, \mathcal{T}^{m}\right)$ is indecomposably finite and has a polynomial description of degree $d=2$. 
6.3. Projective space. In this subsection we prove part (iii) of Theorem 1.11, Let $X=\mathbb{P}_{k}^{d}=$ Proj $k\left[z_{0}, z_{1}, \ldots, z_{d}\right]$. We assume that $G$ acts on $\mathbb{P}_{k}^{d}$ over $k$ and that this action is faithful and generically free such that $\mathcal{L}=\mathcal{O}(1)$ is a (very ample) $G$ equivariant line bundle on $\mathbb{P}_{k}^{d}$. By Lemma 4.3, we can assume $k$ is an algebraically closed field of characteristic $p>0$. After a linear change of the homogeneous coordinates $z_{0}, \ldots, z_{d}$, we may assume that relative to these coordinates the action of elements in a Sylow $p$-subgroup $G_{p}$ of $G$ on $\mathrm{H}^{0}(\mathcal{L})=k z_{0}+\cdots+k z_{d}$ is given by upper triangular unipotent matrices. Define $T$ to be the hyperplane $z_{0}=0$, and $Q$ to be the point $(0: \cdots: 0: 1)$ in $\mathbb{P}_{k}^{d}$ over $k$. Then both $T$ and $Q$ are $G_{p}$-stable. Let $Y=\mathbb{P}_{k}^{d} / G$, define $\pi: \mathbb{P}_{k}^{d} \rightarrow Y$ to be the quotient morphism, and let $b$ (resp. $B$ ) be the branch locus in $Y$ (resp. the ramification locus in $\mathbb{P}_{k}^{d}$ ) of $\pi$.

Our goal is to construct a complex with $d+2$ terms which is an exact Koszul resolution, and then to study its splitting behavior.

Definition 6.3.1. By Lemma 6.1.3, there exist effective Weil divisors $D_{0}, \ldots, D_{d-1}$ on $Y$ such that when $D_{j}^{\prime}=\pi^{*}\left(D_{j}\right)$ the following is true:

(a) for all $j, D_{j}^{\prime}$ does not contain any point in the $G$-orbit of $Q=(0: \cdots: 0: 1)$;

(b) there is a $G$-equivariant $\mathcal{O}_{\mathbb{P}_{k}^{d}}$-module isomorphism,

$$
\lambda: \quad \mathcal{L}^{m} \rightarrow \mathcal{O}_{\mathbb{P}_{k}^{d}}\left(D_{0}^{\prime}\right),
$$

where $m=m_{2}^{2}(\# G)$ and $m_{2}$ is the maximal prime to $p$ divisor of \#G;

(c) the divisors $D_{0}, \ldots, D_{d-1}$ are all linearly equivalent on $Y$;

(d) for each subset $J \subset\{0, \ldots, d-1\}$ and each point $x \in \bigcap_{j \in J} D_{j}^{\prime}$, the local equations of the $D_{j}^{\prime}$ in $\mathcal{O}_{\mathbb{P}_{k}^{d}, x}$ for $j \in J$ form a regular sequence;

(e) the intersection $D_{0}^{\prime} \cap \cdots \cap D_{d-1}^{\prime} \cap B=\emptyset$.

Let $D=D_{0}, D^{\prime}=D_{0}^{\prime}$ and let $\mathcal{L}_{D^{\prime}}=\mathcal{O}_{\mathbb{P}_{k}^{d}}\left(D^{\prime}\right)$. Define $f_{0}=1$, and let $f_{1}, f_{2}, \ldots, f_{d-1}$ be in $k(Y)$ with $\operatorname{div}_{Y}\left(f_{j}\right)=D_{j}-D_{0}$. The Koszul resolution for $\mathcal{O}_{D_{0}^{\prime} \cap D_{1}^{\prime} \cap \cdots \cap D_{d-1}^{\prime}}$ has the following form:

$$
0 \rightarrow K_{d} \stackrel{\rho_{d}}{\longrightarrow} K_{d-1} \stackrel{\rho_{d-1}}{\longrightarrow} \cdots \stackrel{\rho_{2}}{\longrightarrow} K_{1} \stackrel{\rho_{1}}{\longrightarrow} K_{0} \stackrel{\rho_{0}}{\longrightarrow} \mathcal{O}_{D_{0}^{\prime} \cap D_{1}^{\prime} \cap \cdots \cap D_{d-1}^{\prime}} \rightarrow 0,
$$

where $K_{0}=\mathcal{O}_{\mathbb{P}_{k}^{d}}$,

$$
K_{1}=\mathcal{O}_{\mathbb{P}_{k}^{d}}\left(-D_{0}^{\prime}\right) \oplus \mathcal{O}_{\mathbb{P}_{k}^{d}}\left(-D_{1}^{\prime}\right) \oplus \cdots \oplus \mathcal{O}_{\mathbb{P}_{k}^{d}}\left(-D_{d-1}^{\prime}\right)=\bigoplus_{0 \leq i \leq d-1} f_{i} \mathcal{L}_{D^{\prime}}^{-1},
$$

and, for $2 \leq r \leq d$,

$$
K_{r}=\bigwedge^{r} K_{1}=\bigoplus_{0 \leq i_{1}<i_{2}<\cdots<i_{r} \leq d-1} f_{i_{1}} f_{i_{2}} \cdots f_{i_{r}} \mathcal{L}_{D^{\prime}}^{-r}
$$

The morphisms $\rho_{1}$ and $\rho_{0}$ are the obvious ones, and for $2 \leq r \leq d, \rho_{r}$ is defined by "contraction," namely

$$
\rho_{r}\left(t_{1} \wedge \cdots \wedge t_{r}\right)=\sum_{j=1}^{r}(-1)^{j-1} \rho_{1}\left(t_{j}\right)\left(t_{1} \wedge \cdots \wedge \widehat{t_{j}} \wedge \cdots \wedge t_{r}\right) .
$$

Because of property (d) above the Koszul resolution is exact (see [21, Thm. 43, p. 135]). Tensoring the Koszul resolution with any positive power of $\mathcal{L}$ results in an exact sequence. Since $\mathcal{L}$ is very ample, there exists an integer $\mu_{0} \geq d$ such that for all $0 \leq r \leq d, \mathrm{H}^{1}\left(\operatorname{ker}\left(\rho_{r}\right) \otimes \mathcal{L}^{n}\right)=0$ for all $n \geq m \mu_{0}$. Now let $j$ be an integer with $0 \leq j<m$, and let $t \geq \mu_{0}$ be an integer. The isomorphism 
$\lambda: \mathcal{L}^{m} \rightarrow \mathcal{O}_{\mathbb{P}_{k}^{d}}\left(D_{0}^{\prime}\right)=\mathcal{O}_{\mathbb{P}_{k}^{d}}\left(D^{\prime}\right)$ gives an isomorphism $\lambda^{\otimes t}: \mathcal{L}^{t m+j} \rightarrow \mathcal{L}_{D^{\prime}}^{t} \otimes \mathcal{L}^{j}$. By tensoring the Koszul resolution (6.17) with $\mathcal{L}_{D^{\prime}}^{t} \otimes \mathcal{L}^{j}=\lambda^{\otimes t} \mathcal{L}^{t m+j}$ and taking cohomology on $\mathbb{P}_{k}^{d}$ of the resulting complex, we obtain the following exact Koszul complex of $k G$-modules, denoted by $C_{\bullet}=C_{\bullet}(t, j)$,

$$
0 \rightarrow C_{d} \stackrel{\tau_{d}}{\longrightarrow} C_{d-1} \stackrel{\tau_{d-1}}{\longrightarrow} \cdots \stackrel{\tau_{2}}{\longrightarrow} C_{1} \stackrel{\tau_{1}}{\longrightarrow} C_{0} \stackrel{\tau_{0}}{\longrightarrow} Q_{t, j} \rightarrow 0,
$$

where $Q_{t, j}=\mathrm{H}^{0}\left(\left.\mathcal{L}_{D^{\prime}}^{t} \otimes \mathcal{L}^{j}\right|_{D_{0}^{\prime} \cap D_{1}^{\prime} \cap \cdots \cap D_{d-1}^{\prime}}\right), C_{0}=\mathrm{H}^{0}\left(\mathcal{L}_{D^{\prime}}^{t} \otimes \mathcal{L}^{j}\right)$, and, for $1 \leq r \leq d$,

$$
C_{r}=\bigoplus_{0 \leq i_{1}<i_{2}<\cdots<i_{r} \leq d-1} f_{i_{1}} f_{i_{2}} \cdots f_{i_{r}} \mathrm{H}^{0}\left(\mathcal{L}_{D^{\prime}}^{t-r} \otimes \mathcal{L}^{j}\right) .
$$

The morphism $\tau_{0}$ is the canonical projection, and, for $1 \leq r \leq d, \tau_{r}$ is defined by

$$
\tau_{r}\left(f_{i_{1}} f_{i_{2}} \cdots f_{i_{r}} \cdot h\right)=\sum_{j=1}^{r}(-1)^{j-1} f_{i_{j}}\left(f_{i_{1}} \cdots \widehat{f_{i_{j}}} \cdots f_{i_{r}} \cdot h\right)
$$

for $h \in \mathrm{H}^{0}\left(\mathcal{L}_{D^{\prime}}^{t-r} \otimes \mathcal{L}^{j}\right)$.

Note that since $D_{0}^{\prime} \cap D_{1}^{\prime} \cap \cdots \cap D_{d-1}^{\prime}$ is a 0 -dimensional subvariety of $\mathbb{P}_{k}^{d}$ with étale Galois $G$-action, $Q_{t, j}$ is a free $k G$-module by Lemma 6.1.4.

Lemma 6.3.2. Let $\mathcal{L}_{D^{\prime}}=\mathcal{O}_{\mathbb{P}_{k}^{d}}\left(D^{\prime}\right)$. For $x$ a closed point of $X, t \geq \mu_{0}, 0 \leq j<m$ and $1 \leq r \leq d$, let $\mathfrak{m}_{\mathbb{P}_{k}^{d}, x}$ be the maximal ideal of $\mathcal{O}_{\mathbb{P}_{k}^{d}, x}$, and define

$$
s(t, j, r, x)=\frac{\left(\mathcal{L}_{D^{\prime}}^{t-r+1} \otimes \mathcal{L}^{j}\right)_{x}}{\mathfrak{m}_{\mathbb{P}_{k}^{d}, x}^{(t-r) m+j+1} \cdot\left(\mathcal{L}_{D^{\prime}}^{t-r+1} \otimes \mathcal{L}^{j}\right)_{x}} .
$$

Let $H$ be the inertia group of the point $Q=(0: \cdots: 0: 1)$, so that $G_{p} \subset H$. Then for all $0 \leq i \leq d-1$, the natural $k H$-module homomorphism

$$
\sigma_{t-r, j, i, Q}: \quad f_{i} \mathrm{H}^{0}\left(\mathcal{L}_{D^{\prime}}^{t-r} \otimes \mathcal{L}^{j}\right) \quad \rightarrow \quad s(t, j, r, Q)
$$

is an isomorphism.

Proof. Since $\sigma_{t-r, j, i, Q}$ is a $k H$-module homomorphism, it is enough to show it is a $k$-vector space isomorphism. Because $D^{\prime}$ is linearly equivalent to the divisor of $z_{d}^{m}$, we can reduce to showing $\sigma_{t-r, j, i, Q}$ is a $k$-vector space isomorphism when $D^{\prime}$ is the hypersurface $z_{d}^{m}=0$. Since $Q$ does not lie on $z_{d}^{m}=0$, the constant 1 is a local equation for $D^{\prime}$ at $Q$, and $\left(z_{0} / z_{d}\right)$ is a local equation for $T$ at $Q$. We obtain

$$
\begin{aligned}
\frac{\left(\mathcal{L}_{D^{\prime}}^{t-r+1} \otimes \mathcal{L}^{j}\right)_{Q}}{\mathfrak{m}_{\mathbb{P}_{k}^{d}, Q}^{(t-r) m+j+1} \cdot\left(\mathcal{L}_{D^{\prime}}^{t-r+1} \otimes \mathcal{L}^{j}\right)_{Q}} & =\frac{\left(\frac{z_{d}}{z_{0}}\right)^{j} \cdot \mathcal{O}_{\mathbb{P}_{k}^{d}, Q}}{\left(\frac{z_{d}}{z_{0}}\right)^{j} \cdot \mathfrak{m}_{\mathbb{P}_{k}^{d}, Q}^{(t-r) m+j+1}}=\frac{\left(\frac{z_{d}}{z_{0}}\right)^{j} f_{i} \cdot \mathcal{O}_{\mathbb{P}_{k}^{d}, Q}}{\left(\frac{z_{d}}{z_{0}}\right)^{j} f_{i} \cdot \mathfrak{m}_{\mathbb{P}_{k}^{d}, Q}^{(t-r) m+j+1}} \\
& =\left(\frac{z_{d}}{z_{0}}\right)^{j} f_{i} \cdot \frac{\mathcal{O}_{\mathbb{P}_{k}^{d}, Q}}{\mathfrak{m}_{\mathbb{P}_{k}^{d}, Q}^{(t-r) m+j+1}},
\end{aligned}
$$

where the second equation follows since $f_{i}$ is a unit in $\mathcal{O}_{\mathbb{P}_{k}^{d}, Q}$. Then

$$
\frac{\mathcal{O}_{\mathbb{P}_{k}^{d}, Q}}{\mathfrak{m}_{\mathbb{P}_{k}^{d}, Q}^{(t-r) m+j+1}}=\frac{k\left[\frac{z_{0}}{z_{d}}, \ldots, \frac{z_{d-1}}{z_{d}}\right]}{\left(\frac{z_{0}}{z_{d}}, \ldots, \frac{z_{d-1}}{z_{d}}\right)^{(t-r) m+j+1}},
$$

and the latter module can be identified with the space of all polynomials in $\frac{z_{0}}{z_{d}}, \ldots$, $\frac{z_{d-1}}{z_{d}}$ of degree $\leq(t-r) m+j$. Now let $h \in \mathrm{H}^{0}\left(\mathcal{L}_{D^{\prime}}^{t-r} \otimes \mathcal{L}^{j}\right)$. This means that 
$\operatorname{div}_{X}(h)+(t-r) D^{\prime}+j T \geq 0$, i.e. $h=g /\left(z_{d}^{(t-r) m} z_{0}^{j}\right)$, where $g$ is a homogeneous polynomial in $k\left[z_{0}, z_{1}, \ldots, z_{d}\right]$ of degree $(t-r) m+j$. Then

$$
f_{i} h=\left(\frac{z_{d}}{z_{0}}\right)^{j} f_{i} \cdot \frac{g}{z_{d}^{(t-r) m+j}}
$$

and $\frac{g}{z_{d}^{(t-r) m+j}}$ can be identified with a polynomial in $\frac{z_{0}}{z_{d}}, \ldots, \frac{z_{d-1}}{z_{d}}$ of degree $\leq$ $(t-r) m+j$. Also, as $h$ runs over all the elements in $\mathrm{H}^{0}\left(\mathcal{L}_{D^{\prime}}^{t-r} \otimes \mathcal{L}^{j}\right)$, all polynomials in $\frac{z_{0}}{z_{d}}, \ldots, \frac{z_{d-1}}{z_{d}}$ of degree $\leq(t-r) m+j$ occur. Hence the morphism $\sigma_{t-r, j, i, Q}$ from $f_{i} \mathrm{H}^{0}\left(\mathcal{L}_{D^{\prime}}^{t-r} \otimes \mathcal{L}^{j}\right)$ to $\frac{\left(\mathcal{L}_{D^{\prime}}^{t-r+1} \otimes \mathcal{L}^{j}\right)_{Q}}{\mathfrak{m}_{\mathbb{P}_{k}^{d}, Q}^{(t-r) m+j+1} \cdot\left(\mathcal{L}_{D^{\prime}}^{t-r+1} \otimes \mathcal{L}^{j}\right)_{Q}}$ is an isomorphism of $k$-vector spaces.

We have the following corollary from Lemma 6.3 .2 and the fact that every finitely generated $k G$-module is $(G, H)$-injective when $G_{p} \subset H$ [6, Thm. 19.2 and Prop. 19.5].

Corollary 6.3.3. Suppose $\mathcal{L}_{D^{\prime}}, t, j, r, Q, H$ and $i$ are as in Lemma 6.3.2. Let $\omega(Q)$ be the $G$-orbit of $Q$. The $\sigma_{t-r, j, i, Q^{\prime}}$ over $Q^{\prime} \in \omega(Q)$ give a split injection

$$
\sigma_{t-r, j, i}: \quad f_{i} \mathrm{H}^{0}\left(\mathcal{L}_{D^{\prime}}^{t-r} \otimes \mathcal{L}^{j}\right) \rightarrow \bigoplus_{Q^{\prime} \in \omega(Q)} s\left(t, j, r, Q^{\prime}\right)=s(t, j, r)
$$

of $k G$-modules. Let $\tau: s(t, j, r) \rightarrow f_{i} \mathrm{H}^{0}\left(\mathcal{L}_{D^{\prime}}^{t-r} \otimes \mathcal{L}^{j}\right)$ be a splitting homomorphism. The natural restriction homomorphisms $\mathrm{H}^{0}\left(\mathcal{L}_{D^{\prime}}^{t-r+1} \otimes \mathcal{L}^{j}\right) \rightarrow\left(\mathcal{L}_{D^{\prime}}^{t-r+1} \otimes \mathcal{L}^{j}\right)_{Q^{\prime}}$ associated to $Q^{\prime} \in \omega(Q)$ give a $k G$-module homomorphism

$$
\mathrm{H}^{0}\left(\mathcal{L}_{D^{\prime}}^{t-r+1} \otimes \mathcal{L}^{j}\right) \quad \rightarrow \quad s(t, j, r)
$$

Let

$$
\widehat{\sigma}_{t-r, j}: \quad \mathrm{H}^{0}\left(\mathcal{L}_{D^{\prime}}^{t-r+1} \otimes \mathcal{L}^{j}\right) \quad \rightarrow \quad f_{i} \mathrm{H}^{0}\left(\mathcal{L}_{D^{\prime}}^{t-r} \otimes \mathcal{L}^{j}\right)
$$

be the composition of (6.20) with $\tau$, and let $U_{t-r+1, j}=\operatorname{ker}\left(\widehat{\sigma}_{t-r, j}\right)$. Then we have an internal direct sum as $k G$-modules:

$$
\mathrm{H}^{0}\left(\mathcal{L}_{D^{\prime}}^{t-r+1} \otimes \mathcal{L}^{j}\right)=U_{t-r+1, j} \oplus f_{i} \mathrm{H}^{0}\left(\mathcal{L}_{D^{\prime}}^{t-r} \otimes \mathcal{L}^{j}\right) .
$$

We now prove the following result for $\mathbb{P}_{k}^{2}$ and $\mathbb{P}_{k}^{3}$, which also proves part (iii) of Theorem 1.11

Theorem 6.3.4. Let $d \in\{2,3\}$. Then the Koszul complex $C_{\bullet}(t, j)$ in (6.18) splits completely as a complex of $k G$-modules for all $0 \leq j<m$ and for all $t \geq \mu_{0}$. Moreover, the polynomial ring $k\left[z_{0}, z_{1}, \ldots, z_{d}\right]$ is an indecomposably finite $k G$-module with a polynomial description of degree $d$.

Remark 6.3.5. Suppose $k$ is transcendental over $\mathbb{F}_{p}$. Then already in the case of the projective line $\mathbb{P}_{k}^{1}$ over $k$, there may exist finite group actions which cannot be realized over any algebraic extension of $\mathbb{F}_{p}$. For example, suppose the characteristic of $k$ is 2 and $G=\mathbb{Z} / 2 \times \mathbb{Z} / 2=\langle x, y\rangle$. For each $c \in k$ one has an indecomposable representation $\rho_{c}$ defined by

$$
x \mapsto\left[\begin{array}{ll}
1 & 1 \\
0 & 1
\end{array}\right], \quad y \mapsto\left[\begin{array}{ll}
1 & c \\
0 & 1
\end{array}\right],
$$


and $\rho_{c}$ and $\rho_{c^{\prime}}$ are isomorphic if and only if $c=c^{\prime}$. For this reason, Theorem 6.3.4 does not follow from Karagueuzian's and Symonds' results in [18, 28, in the case $d=3$. However, the case $d=2$ was proved for an arbitrary field $k$ of characteristic $p$ in [19].

To prove Theorem 6.3.4 we will use the following lemma.

Lemma 6.3.6. Suppose

$$
0 \rightarrow M_{n} \stackrel{\delta_{n}}{\longrightarrow} M_{n-1} \stackrel{\delta_{n-1}}{\longrightarrow} \cdots \stackrel{\delta_{2}}{\longrightarrow} M_{1} \stackrel{\delta_{1}}{\longrightarrow} M_{0} \rightarrow 0
$$

is an exact sequence of $k G$-modules for which $M_{0}$ is projective and for which there is an internal direct sum decomposition $M_{i}=\bigoplus_{l=1}^{s(i)} M_{i, l}$ for each $i>1$ having the following property. Let $\pi_{i, l}: M_{i} \rightarrow M_{i, l}$ be the $l^{\mathrm{th}}$ projection and let $\iota_{i, l}: M_{i, l} \rightarrow M_{i}$ be the $l^{\text {th }}$ inclusion. Then if $i>2$, there is an injective map $y_{i}:\{1, \ldots, s(i)\} \rightarrow$ $\{1, \ldots, s(i-1)\}$ such that for all $1 \leq l \leq s(i)$

$$
\lambda_{i, l}=\pi_{i-1, y_{i}(l)} \circ \delta_{i} \circ \iota_{i, l}: M_{i, l} \rightarrow M_{i-1, y_{i}(l)}
$$

is a split injection. Under these conditions, (6.21) splits completely as a sequence of $k G$-modules.

Proof. The result is clear for $n \leq 2$ and if the pair $(n, s(n))=(3,0)$. Hence we use induction on $(n, s(n))$. Let $W_{n-1, y_{n}(s(n))}$ be a complement in $M_{\left.n-1, y_{n}\left(s_{n}\right)\right)}$ for the image of $\lambda_{n, s(n)}$ in (6.22). Define $M_{n-1}^{\prime}$ to be the inner direct sum

$$
M_{n-1}^{\prime}=\bigoplus_{\substack{1 \leq i \leq s(n-1) \\ i \neq y_{n}(s(n))}} M_{n-1, i} \oplus W_{n-1, y_{n}(s(n))},
$$

and let $\iota_{n-1}^{\prime}: M_{n-1}^{\prime} \rightarrow M_{n-1}$ be the natural inclusion. Using the fact that $\lambda_{n, s(n)}$ from (6.22) is a split injection, it follows that there is a short exact sequence of $k G$-modules,

$$
0 \rightarrow \delta_{n} M_{n, s(n)} \hookrightarrow M_{n-1} \stackrel{\zeta_{n-1}^{\prime}}{\longrightarrow} M_{n-1}^{\prime} \rightarrow 0,
$$

such that $\zeta_{n-1}^{\prime} \circ \iota_{n-1}^{\prime}$ is the identity on $M_{n-1}^{\prime}$. Since $M_{n-1}$ is the internal direct sum $M_{n-1}=\delta_{n} M_{n, s(n)} \oplus \iota_{n-1}^{\prime} M_{n-1}^{\prime}$, it follows that $\delta_{n-1}$ and $\delta_{n-1}^{\prime}=\delta_{n-1} \circ \iota_{n-1}^{\prime}$ have the same image, $N_{n-2}$, in $M_{n-2}$. It also follows that $\delta_{n-1}=\delta_{n-1}^{\prime} \circ \zeta_{n-1}^{\prime}$. We obtain a commutative diagram

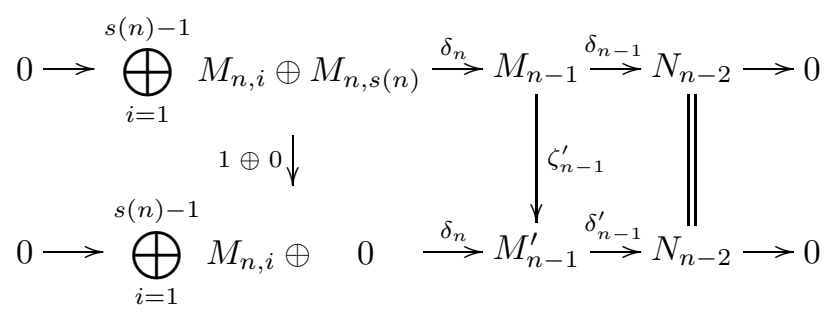

By induction, the bottom row splits; i.e. there is a $k G$-module homomorphism $\alpha_{n-1}^{\prime}: N_{n-2} \rightarrow M_{n-1}^{\prime}$ such that $\delta_{n-1}^{\prime} \circ \alpha_{n-1}^{\prime}$ is the identity on $N_{n-2}$. Then $\alpha_{n-1}=\iota_{n-1}^{\prime} \circ \alpha_{n-1}^{\prime}: N_{n-2} \rightarrow M_{n-1}$ provides a $k G$-module splitting of the top row. By induction, we also get that the exact sequence

$$
0 \rightarrow \bigoplus_{i=1}^{s(n)-1} M_{n, i} \stackrel{\delta_{n}^{\prime}}{\longrightarrow} M_{n-1}^{\prime} \stackrel{\delta_{n-1}^{\prime}}{\longrightarrow} M_{n-2} \stackrel{\delta_{n-2}}{\longrightarrow} \cdots \stackrel{\delta_{2}}{\longrightarrow} M_{1} \stackrel{\delta_{1}}{\longrightarrow} M_{0} \rightarrow 0
$$


splits completely, where $\delta_{n}^{\prime}$ is the restriction of $\delta_{n}$ to the submodule $\bigoplus_{i=1}^{s(n)-1} M_{n, i}$ of $M_{n}$. Hence the splitting of the top row in (6.23) implies that the sequence (6.21) also splits completely.

Proof of Theorem 6.3.4. Let $d=2$ or 3. We first want to use Lemma 6.3.6 to show that the Koszul complex $C_{\bullet}(t, j)$ in (6.18) splits completely as a complex of $k G$-modules for all $0 \leq j<m$ and for all $t \geq \mu_{0}$. When $d=2$, the Koszul complex $C_{\bullet}(t, j)$ is a sequence of the form (6.21) with $n=3, s(3)=1$ and $s(2)=2$. When $d=3, C_{\bullet}(t, j)$ is a sequence of the form with $n=4, s(4)=1$ and $s(3)=3=s(2)$. It follows from Corollary 6.3.3 that for $d=2,3$ the Koszul complex $C_{\bullet}(t, j)$ satisfies the conditions of Lemma 6.3.6. This implies that $C_{\bullet}(t, j)$ splits completely as a complex of $k G$-modules for all $0 \leq j<m$ and for all $t \geq \mu_{0}$.

Hence for all $t \geq \mu_{0}$ and for all $0 \leq j<m$, we have from (6.18) and (6.19) the following equality in $G_{0}^{\oplus}(k G)$ :

$$
\left[Q_{t, j}\right]=\sum_{r=0}^{d}(-1)^{r}\left[C_{r}\right]=\sum_{r=0}^{d}(-1)^{r}\left(\begin{array}{l}
d \\
r
\end{array}\right) \cdot\left[\mathrm{H}^{0}\left(\mathcal{L}^{m(t-r)+j}\right)\right],
$$

where $Q_{t, j}$ is a free $k G$-module. Since $\operatorname{dim}_{k}\left(\mathrm{H}^{0}\left(\mathcal{L}^{m t+j}\right)\right)$ grows as a polynomial of degree $d$ in $t$ for large $t$, this implies $k\left[z_{0}, \ldots, z_{d}\right]=\bigoplus_{n \geq 0} \mathrm{H}^{0}\left(\mathcal{L}^{n}\right)$ is indecomposably finite and has a polynomial description of degree $d$.

\section{REFERENCES}

[1] G. Almkvist and R. Fossum, Decompositions of exterior and symmetric powers of indecomposable $\mathbb{Z} / p \mathbb{Z}$-modules and relations to invariants. In: Séminarie d'Algèbre Paul Dubreil, Proceedings, Paris, 1976-7, Lecture Notes in Mathematics, Vol. 641, Springer-Verlag, BerlinNew York, 1978. MR 499459 (81b:14024)

[2] J. Alperin and L. G. Kovacs, Periodicity of Weyl modules for SL(2, q). J. Algebra 74 (1982), 52-54. MR644217 (83c:20014)

[3] P. Baum, W. Fulton and G. Quart, Lefschetz-Riemann-Roch for singular varieties. Acta Math. 143 (1979), 193-211. MR549774 (82c:14022a)

[4] T. Chinburg and B. Erez, Equivariant Euler-Poincaré characteristics and tameness. Asterisque 209 (1992), 179-194. MR1211011 (94c:14015)

[5] T. Chinburg, B. Erez, G. Pappas and M. Taylor, Tame actions of group schemes: Integrals and Slices. Duke Math. J. 82 (1996), 269-308. MR1387229 (97d:14066)

[6] C. Curtis and I. Reiner, Methods of representation theory I, II. John Wiley \& Sons, 1981, 1987.

[7] I. Dolgachev, Lectures on Invariant Theory. Cambridge University Press, 2003. MR2004511 (2004g:14051)

[8] P. Deligne, Fonctions $L$ modulo $l^{n}$ et modulo $p$. In: Cohomology étale (SGA 4.5) by P. Deligne, with J.-F. Boutot, L. Illusie and J.-L. Verdier, Cohomology. Lecture Notes in Math., Vol. 569, Springer-Verlag, Heidelberg, 1977. MR0463174(57:3132)

[9] D. Edidin and W. Graham, Riemann-Roch for equivariant Chow groups, Duke Math. J. 102 (2000), 567-594. MR:1756110 (2001f:14018)

[10] D. Edidin and W. Graham, Nonabelian localization in equivariant $K$-theory and RiemannRoch for quotients, Adv. Math. 198 (2005), 547-582. MR2183388(2006h:14014)

[11] W. Fulton, Intersection theory. Springer-Verlag, New York-Berlin-Heidelberg, 1984. MR.732620 (85k:14004)

[12] W. Fulton and S. Lang, Riemann-Roch Algebra. Springer-Verlag, New York-BerlinHeidelberg, 1985. MR801033 (88h:14011)

[13] D. J. Glover, A study of certain modular representations. J. Algebra 51 (1978), 425-475. MR0476841(57:16392)

[14] A. Grothendieck, Étude cohomologique des faisceaux coh'erents (EGA3), Publ. Math. IHES Vols. 11 and 17, 1961 and 1963. 
[15] A. Grothendieck, with P. Deligne, P. and N. Katz, Groupes de monodromie en géométrie algébriques (SGA 7), 1967-68. Lecture Notes in Math., Vols. 288, 340, Springer-Verlag, Heidelberg, 1972-1973.

[16] R. Hartshorne, Algebraic geometry. Graduate Texts in Math. 52, Springer-Verlag, Berlin-New York-Heidelberg, 1977. MR0463157 (57:3116)

[17] D. B. Karagueuzian and P. Symonds, The module structure of a group action on a polynomial ring. J. Algebra 218 (1999), 672-692. MR 1705758 (2000f:20011)

[18] D. B. Karagueuzian and P. Symonds, The module structure of a group action on a polynomial ring: A finiteness theorem. Manuscript, 2002, 20 pages.

[19] D. B. Karagueuzian, P. Symonds, The module structure of a group action on a polynomial ring: Examples, generalizations, and applications. In: Invariant Theory in all characteristics, 139-158, CRM Proc. Lecture Notes, 35, AMS, Providence, RI, 2004. MR2066462 (2005g:13011)

[20] B. Köck, The Grothendieck-Riemann-Roch theorem for group scheme actions, Ann. Sci. École Norm. Sup. (4) 31 (1998), 415-458. MR:1621405 (99f:14010)

[21] H. Matsumura, Commutative algebra. $2^{\text {nd }}$ edition, W. A. Benjamin Co., New York, 1980. MR575344 (82i:13003)

[22] J. S. Milne, Étale Cohomology. Princeton Univ. Press, 1980. MR.559531 (81j:14002)

[23] S. Nakajima, On Galois module structure of the cohomology groups of an algebraic variety. Inv. Math. 75 (1984), 1-8. MR.728135 (85j:14021)

[24] G. Pappas, Galois module structure and the $\gamma$-filtration. Compositio Math. 121 (2000), 79104. MR.1753111 (2001f:14032)

[25] G. Pappas, Galois modules, ideal class groups and cubic structures, Preprint (arXiv.org/math.NT/0306309).

[26] J.-P. Serre, Linear representations of finite groups. Graduate Texts in Math. 42, SpringerVerlag, New York, 1977. MR0450380 (56:8675)

[27] J.-P. Serre, Local fields. Graduate Texts in Math. 67, Springer-Verlag, New York, 1979. MR554237 (82e:12016)

[28] P. Symonds, Group actions on polynomial and power series rings. Pacific J. Math. 195 (2000), 225-230. MR 1781621 (2001h:13008)

[29] P. Symonds, Structure theorems over polynomial rings. Manuscript, 2005, 10 pages.

[30] S. V. Ullom, A survey of class groups of integral group rings. In: Algebraic Number Fields (Lfunctions and Galois properties), 497-524, Academic Press, London-New York-San Francisco, 1977.

[31] G. Vezzosi and A. Vistoli, Higher algebraic $K$-theory of group actions with finite stabilizers, Duke Math. J. 113 (2002), 1-55. MR1905391 (2003g:19004)

[32] G. Vezzosi and A. Vistoli, Higher algebraic $K$-theory for actions of diagonalizable groups, Invent. Math. 153 (2003), 1-44. Erratum: Invent. Math. 161 (2005), 219-224. MR1990666 (2006c:19003)

Department of Mathematics, University of Iowa, Iowa City, Iowa 52242-1419

E-mail address: fbleher@math.uiowa.edu

Department of Mathematics, University of Pennsylvania, Philadelphia, Pennsylvania 19104-6395

E-mail address: ted@math.upenn.edu 